\title{
REGULARITY FOR THE NEAR FIELD PARALLEL REFRACTOR AND REFLECTOR PROBLEMS
}

\author{
CRISTIAN E. GUTIÉRREZ AND FEDERICO TOURNIER
}

\begin{abstract}
Авstract. We prove local interior $C^{1, \alpha}$ estimates of solutions for the parallel refractor and reflector problems. Our main assumptions are on the target set $\Sigma$ and no assumptions are made on the smoothness of the densities.
\end{abstract}

Contents

\begin{tabular}{|c|c|}
\hline \multicolumn{2}{|l|}{ 1. Introduction } \\
\hline 2. $\quad$ Preliminaries & 2 \\
\hline 2.1. Refraction & 2 \\
\hline 2.2. $\quad$ Ellipsoids & 3 \\
\hline 2.3. $\quad$ Set up & 4 \\
\hline 2.4. Estimates for the derivatives of $\phi$ & 5 \\
\hline 2.5. Assumptions on the target $\Sigma$ & 7 \\
\hline 3. Regularity hypothesis on the target set $\Sigma$ & 9 \\
\hline 4. Definition of refractor and main results & 14 \\
\hline 4.1. Refractors & 14 \\
\hline 4.2. Local and global refractors & 16 \\
\hline 4.3. Main result & 21 \\
\hline 4.4. A property of refractors & 22 \\
\hline 5. Hölder continuity of the gradient of the refractor & 2 \\
\hline 6. Example of a target for refraction & 2 \\
\hline 7. On the definition of refractor & 2 \\
\hline 8. Regularity results for the parallel reflector problem in the near field case & 2 \\
\hline 8.1. Reflection & 2 \\
\hline 8.2. $\quad$ Set up & 29 \\
\hline 8.3. Hypothesis on the target set & 29 \\
\hline 8.4. Definition of parallel reflector and hypothesis on the measures & \\
\hline 8.5. On the definition of reflector & \\
\hline
\end{tabular}

\section{INTRODUCTION}

Suppose we have a domain $\Omega \subset \mathbb{R}^{n}$ and a domain $\Sigma$ contained in an $n$ dimensional surface in $\mathbb{R}^{n+1} ; \Sigma$ is referred as the target domain or screen to be illuminated. Let $n_{1}$ and $n_{2}$ be the indexes of refraction of two homogeneous and isotropic media I and II, respectively, and suppose that from the region $\Omega$ surrounded by medium $\mathrm{I}$, radiation emanates in the vertical direction $e_{n+1}$ with intensity $f(x)$ for $x \in \Omega$, and $\Sigma$ is surrounded by media II. That is, all emanating rays from $\Omega$ are collimated. A parallel refractor is an optical surface $\mathcal{R}$ interface between media I and II, such

March 18, 2014.

The first author was partially supported by NSF grant DMS-1201401. 
that all rays refracted by $\mathcal{R}$ into medium II are received at the surface $\Sigma$, and the prescribed radiation intensity received at each point $p \in \Sigma$ is $\sigma(p)$. Assuming no loss of energy in this process, we have the conservation of energy equation $\int_{\Omega} f(x) d x=\int_{\Sigma} \sigma(p) d p$. Under general conditions on $\Omega$ and $\Sigma$, and when $\sigma$ is a Radon measure in $D$, the existence of parallel refractors is proved in [?].

The purpose of this paper is to study regularity of parallel refractors and reflectors. Indeed, under suitable conditions on the target and the measure $\sigma$, we prove interior $C^{1, \alpha}$ estimates. We prove that if $u$ is a parallel refractor in $\Omega$, the target $\sum$ satisfies the local condition (3.6) from $\left(x^{\star}, u\left(x^{\star}\right)\right)$, and the measure $\sigma$ satisfies a local condition at that point, condition (5.1), then $u \in C^{1, \alpha}$ in a neighborhood of the point $x^{\star}$.

Throughout the paper we assume that media II is denser than media $I$, that is, $n_{1}<n_{2}$. When $n_{1}>n_{2}$, the geometry of the refractor changes. One needs to use hyperboloids of revolution instead of ellipsoids as indicated in [?].

The plan of the paper is as follows. Section 2 contains results concerning estimates of ellipsoids of revolution, and Subsection 2.5 contains basic assumptions on the target. Section 3 contains assumptions on the target modeled on the conditions introduced by Loeper [?, Proposition 5.1]. Indeed, we assume the target satisfies the local condition (3.1). We also introduce a differential condition (3.2), similar in form to condition (A3) of Ma, Trudinger and Wang [?]; and show in Theorem 3.2 and Remark 3.3 that $(3.6)$ and (3.1) are equivalent. Section 4 contains the main results Lemma 4.2 and Theorem 4.5 , and also Proposition 4.6 used later for the application of these results to show regularity of parallel refractors constructed in [?], Corollary 5.3. Section 5 contains Hölder estimates of gradients of refractors under the assumptions (5.1), (5.2), on the target $\Sigma$ and the measure $\sigma$ on $\Sigma$. Section 6 contains examples of targets verifying the assumptions, see condition (6.3). Up to this point in the paper, refractors are defined with ellipsoids supporting the refractor from above. Refractors can also be defined with ellipsoids supporting from below, and in Section 7 we obtain the same regularity results for refractors with this definition. In Section 8 we obtain similar regularity results for the near field parallel reflector problem.

Acknowledgements. It is a pleasure to thank Neil Trudinger and Philippe Delanoë for useful comments and suggestions.

\section{Preliminaries}

2.1. Refraction. We briefly review the process of refraction. Our setting is $\mathbb{R}^{n+1}$. Points will be denoted by $X=\left(x, x_{n+1}\right)$. We consider parallel rays traveling in the unit direction $e_{n+1}$. Let $T$ be a hyperplane with upper unit normal $N$ and $X \in T$. We assume that the region below $T$ has refractive index $n_{1}$ and the region above $T$ has refractive index $n_{2}$ and $\kappa:=\frac{n_{1}}{n_{2}}<1$, e.g., air to glass. In such case, by Snell's law of refraction, a ray coming from below in direction $e_{n+1}$ that hits $T$ at $X$ is 
refracted in the unit direction

$$
\Lambda=\kappa e_{n+1}+\delta N, \quad \text { with } \delta=-\kappa e_{n+1} \cdot N+\sqrt{1+\kappa^{2}\left(\left(e_{n+1} \cdot N\right)^{2}-1\right)},
$$

where $\delta>0$ since $\kappa<1$.

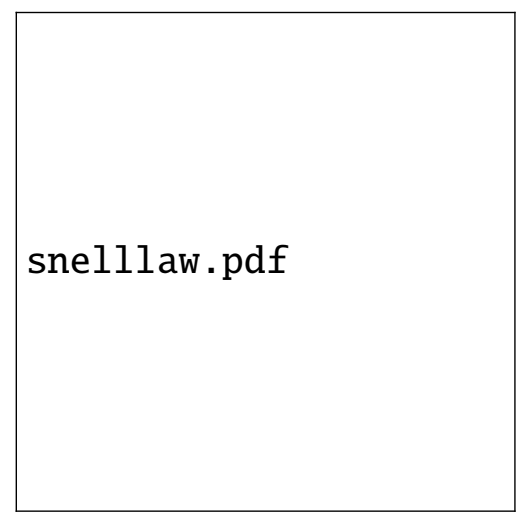

Figure 1. Snell's law, $n_{1}<n_{2}$

In particular, if $v \in \mathbb{R}^{n}$ and the hyperplane $T$ is so that the unit upper normal $N=\frac{(-v, 1)}{\sqrt{1+|v|^{2}}}$, then the refracted unit direction is

$$
\Lambda(v):=\left(-\frac{\delta}{\sqrt{1+|v|^{2}}} v, \frac{\delta}{\sqrt{1+|v|^{2}}}+\kappa\right):=(-Q(v) v, Q(v)+\kappa),
$$

with $Q(v)^{2}|v|^{2}+(Q(v)+\kappa)^{2}=1$ and $Q>q^{7}$. The refracted ray is $X+s \Lambda$, for $s>0$. Here we have in mind that $T$ is the tangent plane to a refractor $u$ at $(x, u(x))$ and so $v=D u(x)$.

2.2. Ellipsoids. Given $b>0$ and $Y=\left(y, y_{n+1}\right)$, consider

$$
E(Y, b)=\left\{X:|X-Y|+k\left(x_{n+1}-y_{n+1}\right)=b\right\} .
$$

$E(Y, b)$ is an ellipsoid of revolution with foci $\left(y, y_{n+1}\right),\left(y, y_{n+1}-\frac{2 \kappa b}{1-\kappa^{2}}\right)$. The projection of $E(Y, b)$ over $\mathbb{R}^{n}$ is the ball $B_{b / \sqrt{1-\kappa^{2}}}(y)$. We define the lower and upper parts of $E(Y, b)$ by

$$
\begin{aligned}
& E^{-}(Y, b)=\left\{X \in E(Y, b): x_{n+1} \leq y_{n+1}-\frac{\kappa b}{1-\kappa^{2}}\right\}, \\
& E^{+}(Y, b)=\left\{X \in E(Y, b): x_{n+1} \geq y_{n+1}-\frac{\kappa b}{1-\kappa^{2}}\right\},
\end{aligned}
$$

"We have in this case, $\delta=\frac{-\kappa+\sqrt{1+\left(1-\kappa^{2}\right)|v|^{2}}}{\sqrt{1+|v|^{2}}}$. 
respectively. We can regard $E^{-}(Y, b)$ as the graph of the function

$$
\phi(x)=y_{n+1}-\frac{\kappa b}{1-k^{2}}-\sqrt{\frac{b^{2}}{\left(1-k^{2}\right)^{2}}-\frac{|x-y|^{2}}{1-k^{2}}}
$$

for $x \in B_{b / \sqrt{1-\kappa^{2}}}(y)$. If $X=(x, \phi(x)), x_{n+1}=\phi(x)$, then $D_{x} \phi(x)=\frac{x-y}{y_{n+1}-x_{n+1}-k|X-Y|}$. A ray with direction $e_{n+1}$ that hits from below the graph of $\phi$ at the point $X=(x, \phi(x))$ is refracted along the ray $X+s \Lambda(v)$ with $v=D \phi(x)$, and therefore it passes through the focus $Y$.

If $X=(x, \phi(x))$ and the focus $Y$ can be written as $Y=X+s \Lambda(v)$ for some $s>0$ and $v \in \mathbb{R}^{n}$, then $v=D \phi(x)$.

Given $X, Y \in \mathbb{R}^{n+1}$, let us define

$$
c(X, Y)=|X-Y|+k\left(x_{n+1}-y_{n+1}\right)
$$

and we have

$$
(1-\kappa)|X-Y| \leq c(X, Y) \leq(1+\kappa)|X-Y|
$$

Given $X_{0}, Y \in \mathbb{R}^{n+1}$ with $X_{0} \neq Y$, then $E\left(Y, c\left(X_{0}, Y\right)\right)$ is the unique ellipsoid passing through $X_{0}$ and having upper focus at $Y$.

We have that

$$
X \in E^{-}(Y, b) \text { iff } y_{n+1}-x_{n+1}-\kappa|X-Y| \geq 0 \text {. }
$$

and

$$
X \in E^{+}(Y, b) \text { iff } y_{n+1}-x_{n+1}-\kappa|X-Y| \leq 0 .
$$

2.3. Set up. We fix a bounded domain $\Omega \subset \mathbb{R}^{n}$ and a cylinder set $C_{\Omega}=\Omega \times(0, M)$; points in $C_{\Omega}$ are denoted by the letter $X$.

Given $0<\delta<1$ we define the region

$\mathcal{T}=\left\{Y \in \mathbb{R}^{n+1}:\right.$ for each $X_{0} \in C_{\Omega}$ we have $X_{0} \in E^{-}\left(Y, c\left(X_{0}, Y\right)\right)$ and $\left.\Omega \subset B_{\delta c\left(X_{0}, Y\right) / \sqrt{1-\kappa^{2}}}(y)\right\}$.

The region $\mathcal{T}$ is open and unbounded. Notice that if $\left(y, y_{n+1}\right) \in \mathcal{T}$, then $\left(y, y_{n+1}^{\prime}\right) \in$ $\mathcal{T}$, for all $y_{n+1}^{\prime} \geq y_{n+1}$. Also, if $Y \in \mathcal{T}$ and $X_{0} \in C_{\Omega}$, then $c\left(X_{0}, Y\right) \geq c_{n}|\Omega|^{1 / n} \sqrt{1-\kappa^{2}} / \delta$ and so from $(2.4$ ) we get

$$
\operatorname{dist}\left(\mathcal{T}, C_{\Omega}\right) \geq C(\delta, \kappa,|\Omega|, n)>0 .
$$

We have that the function

$$
\phi\left(x, Y, X_{0}\right)=y_{n+1}-\frac{k c\left(X_{0}, Y\right)}{1-k^{2}}-\sqrt{\frac{c\left(X_{0}, Y\right)^{2}}{\left(1-k^{2}\right)^{2}}-\frac{|x-y|^{2}}{1-k^{2}}},
$$

$X_{0}=\left(x_{0}, x_{0_{n+1}}\right)$, defines the lower part of the ellipsoid $E\left(Y, c\left(X_{0}, Y\right)\right)$ for $x \in B_{c\left(X_{0}, Y\right) / \sqrt{1-\kappa^{2}}}(y)$. We then have for $X_{0} \in C_{\Omega}, Y \in \mathcal{T}$ and $X \in E^{-}\left(Y, c\left(X_{0}, Y\right)\right), X=\left(x, x_{n+1}\right)$, with $x \in \Omega$ that

$$
y_{n+1}-x_{n+1}-\kappa|X-Y| \geq \beta c(X, Y),
$$


with $\beta=\sqrt{1-\delta^{2}}$.

2.4. Estimates for the derivatives of $\phi$. We show that the function $\phi\left(x, Y, X_{0}\right)$ is differentiable of any order in all variables for $x \in \Omega, Y \in \mathcal{T}$, and $X_{0} \in C_{\Omega}$. Let us calculate first $\frac{\partial \phi}{\partial x_{i}}, 1 \leq i \leq n$. We have

$$
\frac{\partial \phi}{\partial x_{i}}\left(x, Y, X_{0}\right)=\frac{1}{1-\kappa^{2}} \frac{x_{i}-y_{i}}{\sqrt{\frac{c\left(X_{0}, Y\right)^{2}}{\left(1-k^{2}\right)^{2}}-\frac{|x-y|^{2}}{1-k^{2}}}} .
$$

We have $c(X, Y)=|X-Y|+\kappa\left(x_{n+1}-y_{n+1}\right)=c\left(X_{0}, Y\right)$, with $X=\left(x, \phi\left(x, Y, X_{0}\right)\right), x \in \Omega$, then

$$
\begin{aligned}
y_{n+1}-x_{n+1}-\kappa|X-Y| & =y_{n+1}-x_{n+1}-\kappa c\left(X_{0}, Y\right)+\kappa^{2}\left(x_{n+1}-y_{n+1}\right) \\
& =\left(1-\kappa^{2}\right)\left(y_{n+1}-x_{n+1}\right)-\kappa c\left(X_{0}, Y\right) \\
& =\left(1-\kappa^{2}\right)\left(\frac{\kappa c\left(X_{0}, Y\right)}{1-k^{2}}+\sqrt{\frac{c\left(X_{0}, Y\right)^{2}}{\left(1-\kappa^{2}\right)^{2}}-\frac{|x-y|^{2}}{1-k^{2}}}\right)-\kappa c\left(X_{0}, Y\right) \\
& =\left(1-\kappa^{2}\right) \sqrt{\frac{c\left(X_{0}, Y\right)^{2}}{\left(1-\kappa^{2}\right)^{2}}-\frac{|x-y|^{2}}{1-k^{2}}} \geq \sqrt{1-\delta^{2}} c\left(X_{0}, Y\right),
\end{aligned}
$$

for all $x \in \Omega$ from (2.8). Therefore

$$
\left|\frac{\partial \phi}{\partial x_{i}}\left(x, Y, X_{0}\right)\right| \leq \frac{1}{\sqrt{1-\delta^{2}}} \frac{\left|x_{i}-y_{i}\right|}{c\left(X_{0}, Y\right)} .
$$

Let us now calculate $\frac{\partial^{2} \phi}{\partial x_{i} \partial x_{j}}\left(x, Y, X_{0}\right), 1 \leq i, j \leq n$. We have

$$
=\frac{\delta_{i j} \sqrt{\frac{c\left(X_{0}, Y\right)^{2}}{\left(1-k^{2}\right)^{2}}-\frac{|x-y|^{2}}{1-k^{2}}}+\frac{x_{i}-y_{i}}{1-\kappa^{2}} \frac{x_{j}-y_{j}}{\sqrt{\frac{c\left(X_{0}, Y\right)^{2}}{\left(1-k^{2}\right)^{2}}-\frac{|x-y|^{2}}{1-k^{2}}}}}{\left(1-\kappa^{2}\right)\left(\sqrt{\frac{c\left(X_{0}, Y\right)^{2}}{\left(1-k^{2}\right)^{2}}-\frac{|x-y|^{2}}{1-k^{2}}}\right)^{2}} .
$$


So

$$
\begin{aligned}
\left|\frac{\partial^{2} \phi}{\partial x_{i} \partial x_{j}}\left(x, Y, X_{0}\right)\right| & \leq \frac{1}{\left(1-\kappa^{2}\right) \sqrt{\frac{c\left(X_{0}, Y\right)^{2}}{\left(1-k^{2}\right)^{2}}-\frac{|x-y|^{2}}{1-k^{2}}}}+\frac{|x-y|^{2}}{\left(1-\kappa^{2}\right)^{2}\left(\sqrt{\frac{c\left(X_{0}, Y\right)^{2}}{\left(1-k^{2}\right)^{2}}-\frac{|x-y|^{2}}{1-k^{2}}}\right)^{3}} \\
& \leq \frac{1}{\sqrt{1-\delta^{2}} c\left(X_{0}, Y\right)}+\frac{\left(1-\kappa^{2}\right)|x-y|^{2}}{\left(\sqrt{1-\delta^{2}} c\left(X_{0}, Y\right)\right)^{3}} \leq \frac{C(\kappa, \delta)}{c\left(X_{0}, Y\right)} \leq C
\end{aligned}
$$

for $x \in \Omega, Y \in \mathcal{T}$ and $X_{0} \in C_{\Omega}$ by (2.7). Next we estimate the derivatives $\frac{\partial^{2} \phi}{\partial x_{i} \partial y_{j}}\left(x, Y, X_{0}\right), 1 \leq i \leq n, 1 \leq j \leq n+1$. We have

$$
\begin{aligned}
& \frac{\partial^{2} \phi}{\partial x_{i} \partial y_{j}}\left(x, Y, X_{0}\right)=\frac{1}{1-k^{2}} \frac{-\delta_{i j} \sqrt{\frac{c\left(X_{0}, Y\right)^{2}}{\left(1-k^{2}\right)^{2}}-\frac{|x-y|^{2}}{1-k^{2}}}-\left(x_{i}-y_{i}\right) \partial_{y_{j}}\left(\sqrt{\frac{c\left(X_{0}, Y\right)^{2}}{\left(1-k^{2}\right)^{2}}-\frac{|x-y|^{2}}{1-k^{2}}}\right)}{\left(\sqrt{\frac{c\left(X_{0}, Y\right)^{2}}{\left(1-k^{2}\right)^{2}}-\frac{|x-y|^{2}}{1-k^{2}}}\right)^{2}} \\
& -\delta_{i j} \sqrt{\frac{c\left(X_{0}, Y\right)^{2}}{\left(1-k^{2}\right)^{2}}-\frac{|x-y|^{2}}{1-k^{2}}-\left(x_{i}-y_{i}\right)\left(\frac{c\left(X_{0}, Y\right) \frac{\partial c}{\partial y_{j}}\left(X_{0}, Y\right)}{\sqrt{\frac{c\left(X_{0}, Y\right)^{2}}{\left(1-k^{2}\right)^{2}}-\frac{|x-y|^{2}}{1-k^{2}}}}\right)} .
\end{aligned}
$$




$$
\begin{aligned}
\text { Now } \frac{\partial c(X, Y)}{\partial y_{j}}= & -\frac{x_{j}-y_{j}}{|X-Y|}-\kappa \delta_{j(n+1)} \text {. Hence } \\
\left|\frac{\partial^{2} \phi}{\partial x_{i} \partial y_{j}}\left(x, Y, X_{0}\right)\right| & \leq \frac{1}{1-\kappa^{2}}\left(\frac{1}{\left.\sqrt{\frac{c\left(X_{0}, Y\right)^{2}}{\left(1-k^{2}\right)^{2}}-\frac{|x-y|^{2}}{1-k^{2}}}+\frac{|x-y|\left(\frac{(1+\kappa) c\left(X_{0}, Y\right)}{\left(1-k^{2}\right)^{2}}+\frac{|x-y|}{1-\kappa^{2}}\right)}{\left(\sqrt{\frac{c\left(X_{0}, Y\right)^{2}}{\left(1-k^{2}\right)^{2}}}-\frac{|x-y|^{2}}{1-k^{2}}\right.}\right)^{2}}\right) \\
& \leq \frac{1 x-y \mid\left(\frac{(1+\kappa) c\left(X_{0}, Y\right)}{1-k^{2}}+|x-y|\right)}{\sqrt{1-\delta^{2}} c\left(X_{0}, Y\right)}+\frac{\left(\sqrt{1-\delta^{2}} c\left(X_{0}, Y\right)\right)^{2}}{1} \\
& \leq C(\kappa, \delta)\left(\frac{1}{c\left(X_{0}, Y\right)}+1\right) \leq C
\end{aligned}
$$

for $x \in \Omega, Y \in \mathcal{T}$ and $X_{0} \in C_{\Omega}$ by (2.7).

Therefore, we obtain $\left|\frac{\partial^{2} \phi}{\partial x_{i} \partial y_{j}}\left(x, Y, X_{0}\right)\right| \leq C(\delta, K, M,|\Omega|, n)$ for all $x \in \Omega, X_{0} \in C_{\Omega}$, and $Y \in K$. Moreover, these estimates also hold for any $X_{0}$ such that there exists $\bar{X} \in C_{\Omega}$ with $c\left(X_{0}, Y\right)=c(\bar{X}, Y)$.

Continuing in this manner, we get that the function $\phi\left(x, Y, X_{0}\right)$ is $C^{\infty}$ in all its $3 n+2$ variables on $\Omega \times \mathcal{T} \times C_{\Omega}$ and $\left|D^{\alpha} \phi\left(x, Y, X_{0}\right)\right| \leq C$, for any multi-index $\alpha=\left(\alpha_{1}, \ldots, \alpha_{3 n+2}\right)$ with a constant $C$ depending only on $\delta, \kappa, M,|\alpha|$ and $|\Omega|$.

2.5. Assumptions on the target $\Sigma$. We will frequently use the following fact:

(2.11) if the upper focus $Y$ of the ellipsoid defined by $\phi\left(x, Y, X_{0}\right)$ satisfies

$$
Y=X_{0}+s \Lambda(v) \text { for some } s>0 \text { and } v \in \mathbb{R}^{n} \text {, then } D_{x} \phi\left(x_{0}, Y, X_{0}\right)=v \text {. }
$$

Here $\Lambda(v)$ is the unit vector given by (2.1).

We assume the convex hull of the target $\Sigma$ is contained in $\mathcal{T}$, where $\mathcal{T}$ is given by (2.6). For each fixed $X \in C_{\Omega}$, we assume each $Y \in \Sigma$ can be represented parametrically with respect to $X$ by the equation $Y=X+s_{X}(\Lambda) \Lambda$, with $|\Lambda|=1$, where the function $s_{X}$ varies with the point $X$, and $s_{X}(\Lambda)$ is Lipschitz in $\Lambda$ for each $X \in C_{\Omega}$.

Lemma 2.1. For each $X_{0} \in C_{\Omega}$, there exists a constant $C=C\left(X_{0}\right) \geq 1$ such that if $\bar{Y}, \hat{Y} \in \Sigma$ are such that there exist $\bar{v}, \hat{v} \in R^{n}$ and $\bar{s}, \hat{s}>0$ with $\bar{Y}=X_{0}+\bar{s} \Lambda(\bar{v})$, and $\hat{Y}=X_{0}+\hat{s} \Lambda(\hat{v})$, with $\Lambda$ defined by (2.1), then

$$
\frac{1}{C}|\bar{Y}-\hat{Y}| \leq|\bar{v}-\hat{v}| \leq C|\bar{Y}-\hat{Y}| .
$$


This implies that if $\bar{Y} \neq \hat{Y}$, are both in $\Sigma$, then the points $\bar{Y}, \hat{Y}, X_{0}$ cannot be aligned, in other words, from each point $X_{0}$ one can see at most a point in $\Sigma$ on any straight line from $X_{0}$.

Proof. Since the function $s_{X_{0}}(\Lambda)$ is Lipschitz in $\Lambda$, then the left inequality in (2.12) follows. Indeed,

$$
\begin{aligned}
|\bar{Y}-\hat{Y}| & =\left|s_{X_{0}}(\Lambda(\bar{v})) \Lambda(\bar{v})-s_{X_{0}}(\Lambda(\hat{v})) \Lambda(\hat{v})\right| \\
& \leq\left|s_{X_{0}}(\Lambda(\bar{v})) \Lambda(\bar{v})-s_{X_{0}}(\Lambda(\bar{v})) \Lambda(\hat{v})\right|+\left|s_{X_{0}}(\Lambda(\bar{v})) \Lambda(\hat{v})-s_{X_{0}}(\Lambda(\hat{v})) \Lambda(\hat{v})\right| \\
& \leq\left|s_{X_{0}}(\Lambda(\bar{v}))\right||\Lambda(\bar{v})-\Lambda(\hat{v})|+\left|s_{X_{0}}(\Lambda(\bar{v}))-s_{X_{0}}(\Lambda(\hat{v}))\right| .
\end{aligned}
$$

To show the right inequality in (2.12), from (2.11) we can write that $\left(x_{0} \in \Omega\right)$

$$
\begin{aligned}
\left|\bar{v}_{i}-\hat{v}_{i}\right| & =\left|D_{i} \phi\left(x_{0}, \bar{Y}, X_{0}\right)-D_{i} \phi\left(x_{0}, \hat{Y}, X_{0}\right)\right| \\
& =\left|\frac{x_{0_{i}}-\bar{y}_{i}}{\bar{y}_{n+1}-x_{0_{n+1}}-k\left|X_{0}-\bar{Y}\right|}-\frac{x_{0_{i}}-\hat{y}_{i}}{\hat{y}_{n+1}-x_{0_{n+1}}-k\left|X_{0}-\hat{Y}\right|}\right|,
\end{aligned}
$$

$1 \leq i \leq n$

We write

$$
D_{i} \phi\left(x_{0}, \bar{Y}, X_{0}\right)-D_{i} \phi\left(x_{0}, \hat{Y}, X_{0}\right)=D_{Y}\left(D_{i} \phi\right)\left(x_{0}, \tilde{Y}, X_{0}\right) \cdot(\bar{Y}-\hat{Y})
$$

with $\tilde{Y}$ on the segment $[\bar{Y}, \hat{Y}]$ joining $\bar{Y}$ and $\hat{Y}$. This segment is contained in the convex hull of $\Sigma$, and if this convex hull is bounded and contained in $\mathcal{T}$, then the desired estimate follows from the estimates for the derivatives of $\phi$ proved in Subsection (2.4).

The following two lemmas are a consequence of the inequalities for the derivatives of $\phi$.

Lemma 2.2. Let $\bar{X} \in C_{\Omega}, Y \in \mathcal{T}$ and $x_{0} \in \Omega$. Let $x_{0_{n+1}}=\phi\left(x_{0}, Y, \bar{X}\right)$ and set $X_{0}=$ $\left(x_{0}, x_{0_{n+1}}\right)$. Assume that $X_{0}^{\star}=\left(x_{0}, x_{0_{n+1}}-h\right) \in C_{\Omega}$. Then there is a constant $C>0$ such that

for all $x \in \Omega$

$$
0 \leq \phi\left(x, Y, X_{0}\right)-\phi\left(x, Y, X_{0}^{\star}\right) \leq C h,
$$

Proof. We have $0 \leq \phi\left(x, Y, X_{0}\right)-\phi\left(x, Y, X_{0}^{\star}\right)=\frac{\partial \phi}{\partial x_{0_{n+1}}}\left(x, Y, \bar{X}_{0}\right) h$ for some $\bar{X}_{0} \in$ $\left[X_{0}^{\star}, X_{0}\right]$. Since $c\left(\bar{X}_{0}, Y\right)=c(\hat{X}, Y)$ for some $\hat{X} \in C_{M}$, it follows from the estimates in Subsection (2.4) that $\left|\frac{\partial \phi}{\partial x_{0_{n+1}}}\left(x, Y, \bar{X}_{0}\right)\right| \leq C$ for all $x \in \Omega$, where $C$ depends on $\delta, \kappa, M$ and $|\Omega|$.

Lemma 2.3. There exists a constant $C>0$ such that for all $Y, \bar{Y} \in \mathcal{T}$ with the straight segment $[Y, \bar{Y}] \subset \mathcal{T}$, and $X_{0} \in C_{\Omega}$, we have

$$
\left|\phi\left(x, Y, X_{0}\right)-\phi\left(x, \bar{Y}, X_{0}\right)\right| \leq C\left|x-x_{0}\right||Y-\bar{Y}|
$$


for all $x \in \Omega$, which is assumed convex.

Proof. Since $\phi\left(x_{0}, Y, X_{0}\right)=x_{0_{n+1}}$ for all $Y \in \Sigma$, it follows that $\frac{\partial \phi\left(x_{0}, Y, X_{0}\right)}{\partial y_{j}}=0$. We then write

$$
\begin{aligned}
\phi\left(x, Y, X_{0}\right)-\phi\left(x, \bar{Y}, X_{0}\right) & =\sum_{j=1}^{n+1} \frac{\partial \phi\left(x, \xi, X_{0}\right)}{\partial y_{j}}\left(Y_{j}-\bar{Y}_{j}\right), \quad \text { for some } \xi \in[Y, \bar{Y}] \\
& =\sum_{j=1}^{n+1}\left(\frac{\partial \phi\left(x, \xi, X_{0}\right)}{\partial y_{j}}-\frac{\partial \phi\left(x_{0}, \xi, X_{0}\right)}{\partial y_{j}}\right)\left(Y_{j}-\bar{Y}_{j}\right) \\
& =\sum_{j=1}^{n+1} \sum_{i=1}^{n} \frac{\partial^{2} \phi\left(\zeta_{i}, \xi, X_{0}\right)}{\partial x_{i} \partial y_{j}}\left(x_{i}-x_{0_{i}}\right)\left(Y_{j}-\bar{Y}_{j}\right)
\end{aligned}
$$

for some $\zeta_{i} \in\left[x_{0}, x\right]$. Then the lemma follows from the estimates of the derivatives of $\phi$ proved in Subsection (2.4).

3. Regularity HYPOTHESIS ON THE TARGET SET $\Sigma$

Given $\bar{Y}, \hat{Y} \in \mathcal{T}$ and $X_{0} \in C_{\Omega}$, let $\bar{v}=D_{x} \phi\left(x_{0}, \bar{Y}, X_{0}\right), \hat{v}=D_{x} \phi\left(x_{0}, \hat{Y}, X_{0}\right)$, and $v(\lambda)=(1-\lambda) \bar{v}+\lambda \hat{v}$, with $\lambda \in[0,1]$. We consider the set of points

$$
C\left(X_{0}, \bar{Y}, \hat{Y}\right)=\left\{X_{0}+s \Lambda(v(\lambda)): s>0, \lambda \in[0,1]\right\},
$$

where $\Lambda(v(\lambda))$ is defined by (2.1). This set is a two dimensional wedge-shaped surface, in general non-planar, containing all rays having directions $\Lambda(v(\lambda)), 0 \leq$ $\lambda \leq 1$, emanating from $X_{0}$. The curve describing the tip of the vector $\Lambda(v(\lambda)$ ), pictured in Figure 2, is not contained in the plane generated by the rays with directions $\Lambda(\bar{v})$ and $\Lambda(\hat{v})$. If $Y \in C\left(X_{0}, \bar{Y}, \hat{Y}\right)$, then $X_{0} \in E^{-}\left(Y, c\left(X_{0}, Y\right)\right)$. Hence, if $Y(\lambda)=X_{0}+s \Lambda(v(\lambda)) \in C\left(X_{0}, \bar{Y}, \hat{Y}\right)$, then by (2.11) $D_{x} \phi\left(x_{0}, Y(\lambda), X_{0}\right)=v(\lambda)$.

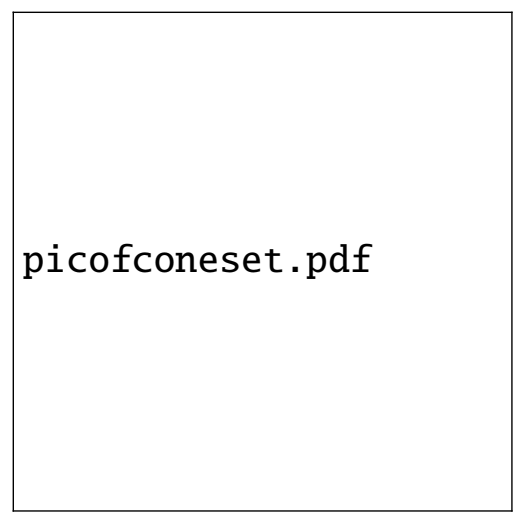

Figure 2. The edges of the wedge are $\Lambda(\bar{v})$ and $\Lambda(\hat{v})$, with $\bar{v}=(1,0)$, $\hat{v}=(-2,1), n_{1}=1, n_{2}=3 / 2$ 
For $\bar{Y}, \hat{Y} \in \mathcal{T}$, and $X_{0} \in C_{\Omega}$, let us define

$$
[\bar{Y}, \hat{Y}]_{X_{0}}:=\Sigma \cap C\left(X_{0}, \bar{Y}, \hat{Y}\right) \text {. }
$$

Notice that by (2.12) each ray $X_{0}+s \Lambda(v(\lambda)) \in C\left(X_{0}, \bar{Y}, \hat{Y}\right)$ intersects $\Sigma$ in at most one point for each $\lambda$. Points in $[\bar{Y}, \hat{Y}]_{X_{0}}$ have the form

$$
Y(\lambda)=X_{0}+s_{X_{0}}(\Lambda(v(\lambda))) \Lambda(v(\lambda)), \quad 0 \leq \lambda \leq 1 .
$$

We will introduce the following local definition on the target $\Sigma$.

Definition 3.1. If $X_{0} \in C_{\Omega}$ we say that the target $\Sigma$ is regular from $X_{0}$ if there exists a neighborhood $U_{X_{0}}$ and positive constants $C_{1}, C_{2}$, depending on $U_{X_{0}}$, such that for all $\bar{Y}, \hat{Y} \in \Sigma$ and $Z=\left(z, z_{n+1}\right) \in U_{X_{0}}$ we have

$$
\phi\left(x, Y_{Z}(\lambda), Z\right) \geq \min \{\phi(x, \bar{Y}, Z), \phi(x, \hat{Y}, Z)\}+C_{1}|\bar{Y}-\hat{Y}|^{2}|x-z|^{2}
$$

for all $x \in \Omega$ with $|x-z| \leq C_{2}, 1 / 4 \leq \lambda \leq 3 / 4$, and $Y_{Z}(\lambda)=Z+s_{Z}(\Lambda(v(\lambda))) \Lambda(v(\lambda))$. Here $\bar{v}=D_{x} \phi(z, \bar{Y}, Z), \hat{v}=D_{x} \phi(z, \hat{Y}, Z)$, and $v(\lambda)=(1-\lambda) \bar{v}+\lambda \hat{v}$.

We introduce below the differential condition (3.2), similar in form to condition (A3) of Ma, Trudinger and Wang [?]. Assuming that the function $s_{X_{0}}$ in the parametrization of the target is $C^{2}$, and the set $[\bar{Y}, \hat{Y}]_{X_{0}}$ is a curve for each $X_{0}$, we prove in the following theorem that such condition is equivalent to (3.1), see also Remark 3.3 for the local case. Theorem 3.2 is similar to a result of Loeper, [?, Proposition 5.1].

Theorem 3.2. Suppose that there exists a constant $C$ such that for all $\xi$ and $\eta$, perpendicular vectors in $R^{n}$, and for $X_{0} \in C_{\Omega}$ and for $Y_{0} \in \Sigma$, we have

$$
\left.\frac{d^{2}}{d \epsilon^{2}}\left\langle D_{x}^{2} \phi\left(x_{0}, Y_{\epsilon}, X_{0}\right) \eta, \eta\right\rangle\right|_{\epsilon=0} \leq-C|\xi|^{2}|\eta|^{2}
$$

where, as before $v_{0}=D \phi\left(x_{0}, Y_{0}, X_{0}\right)$ and $Y_{\epsilon}=X_{0}+s\left(\Lambda\left(v_{0}+\epsilon \xi\right)\right) \Lambda\left(v_{0}+\epsilon \xi\right)$.

Then there exist structural constants $\sigma$ and $C$ such that for $\bar{Y}, \hat{Y} \in \Sigma$ and $X_{0} \in C_{\Omega}$ we have for $\lambda \in[1 / 4,3 / 4]$ and for $\left|x-x_{0}\right| \leq \sigma$ that

$$
\phi\left(x, Y(\lambda), X_{0}\right) \geq \min \left\{\phi\left(x, \bar{Y}, X_{0}\right), \phi\left(x, \hat{Y}, X_{0}\right)\right\}+C|\bar{Y}-\hat{Y}|^{2}\left|x-x_{0}\right|^{2} .
$$

Conversely, (3.3) implies (3.2).

Proof. Let $\bar{Y}, \hat{Y} \in \Sigma$ and $X_{0} \in C_{\Omega}$. Let $v_{\lambda}=(1-\lambda) D \phi\left(x_{0}, \bar{Y}, X_{0}\right)+\lambda D \phi\left(x_{0}, \hat{Y}, X_{0}\right)$ and $\xi=D \phi\left(x_{0}, \hat{Y}, X_{0}\right)-D \phi\left(x_{0}, \bar{Y}, X_{0}\right)$ and $Y(\lambda)=X_{0}+s_{X_{0}}\left(\Lambda\left(v_{\lambda}\right)\right) \Lambda\left(v_{\lambda}\right)$. Notice that for $\eta$ perpendicular to $\xi$ we have

$$
\frac{d^{2}}{d \lambda^{2}}\left\langle D_{x}^{2} \phi\left(x_{0}, Y(\lambda), X_{0}\right) \eta, \eta\right\rangle \leq-C|\xi|^{2}|\eta|^{2},
$$

for all $\lambda \in[0,1]$. Indeed, fix $\lambda \in(0,1)$ and $\xi, \eta \in R^{n}, \xi \perp \eta$. Applying (3.2) with $Y_{0} \rightsquigarrow Y(\lambda), v_{0} \rightsquigarrow v(\lambda):=\bar{v}+\lambda \xi$ (notice that $\bar{v}+\lambda \xi=D_{x} \phi\left(x_{0}, Y(\lambda), X_{0}\right)$ by (2.11)), and $Y_{\epsilon} \rightsquigarrow X_{0}+s_{X_{0}}(\Lambda(v(\lambda)+\epsilon \xi)) \Lambda(v(\lambda)+\epsilon \xi)$, we obtain $\left.\frac{d^{2}}{d \epsilon^{2}}\left\langle D^{2} \phi\left(x_{0}, Y_{\epsilon}, X_{0}\right) \eta, \eta\right\rangle\right|_{\epsilon=0} \leq$ $-C|\xi|^{2}|\eta|^{2}$. Since $Y_{\epsilon}=Y(\epsilon+\lambda)$, the claim follows. 
For $x \in R^{n}$, let $x^{\prime}$ denote the orthogonal projection of $x$ on the hyperplane through $x_{0}$ and normal $\xi$, so $x^{\prime}-x_{0}$ is perpendicular to $\xi$. We let $\eta=x^{\prime}-x_{0}$.

We will first show that there exist positive constants $C_{1}$ and $C_{2}$ such that

$$
\begin{aligned}
& \left\langle D_{x}^{2} \phi\left(x_{0}, Y(\lambda), X_{0}\right)\left(x-x_{0}\right), x-x_{0}\right\rangle \\
& \geq\left\langle\left((1-\lambda) D_{x}^{2} \phi\left(x_{0}, \bar{Y}, X_{0}\right)+\lambda D_{x}^{2} \phi\left(x_{0}, \hat{Y}, X_{0}\right)\right)\left(x-x_{0}\right), x-x_{0}\right\rangle \\
& \quad+\lambda(1-\lambda)|\xi|^{2}\left(C_{1}\left|x-x_{0}\right|^{2}-C_{2}\left|x-x^{\prime}\right|^{2}\right),
\end{aligned}
$$

for all $x \in \Omega$. In fact, fix $x \in \Omega$ and let $f(\lambda)=-\left\langle D_{x}^{2} \phi\left(x_{0}, Y(\lambda), X_{0}\right)\left(x^{\prime}-x_{0}\right), x^{\prime}-x_{0}\right\rangle$. From (3.4), we have $f^{\prime \prime}(\lambda) \geq C\left|x^{\prime}-x_{0}\right|^{2}|\xi|^{2}$. We claim that $f(\lambda) \leq(1-\lambda) f(0)+\lambda f(1)-$ $C\left|x^{\prime}-x_{0}\right|^{2}|\xi|^{2} \lambda(1-\lambda)$ for all $\lambda \in[0,1]$. To prove this claim, fix $\bar{\lambda} \in(0,1)$. By Taylor's theorem, we have that $f(\lambda) \geq f(\bar{\lambda})+f^{\prime}(\bar{\lambda})(\lambda-\bar{\lambda})+C / 2\left|x^{\prime}-x_{0}\right|^{2}|\xi|^{2}(\lambda-\bar{\lambda})^{2}$ for all $\lambda \in[0,1]$. Applying this inequality, first for $\lambda=0$ and then for $\lambda=1$, multiplying the first by $1-\bar{\lambda}$ and the second by $\bar{\lambda}$, and then adding yields $(1-\bar{\lambda}) f(0)+\bar{\lambda} f(1) \geq$ $f(\bar{\lambda})+C\left|x^{\prime}-x_{0}\right|^{2}|\xi|^{2} \bar{\lambda}(1-\bar{\lambda})$ which proves the claim.

Let $g(\lambda)=\left\langle D_{x}^{2} \phi\left(x_{0}, Y(\lambda), X_{0}\right)\left(x^{\prime}-x_{0}\right), x^{\prime}-x_{0}\right\rangle-\left\langle D_{x}^{2} \phi\left(x_{0}, Y(\lambda), X_{0}\right)\left(x-x_{0}\right), x-x_{0}\right\rangle$. Since $s_{X_{0}}$ is $C^{2}$, we have that $\left|\frac{d^{2} D_{i j} \phi\left(x_{0}, Y(\lambda), X_{0}\right)}{d \lambda^{2}}\right| \leq C|\xi|^{2}$ and therefore $\left|g^{\prime \prime}(\lambda)\right| \leq$ $C|\xi|^{2}\left|x-x^{\prime}\right|\left|x-x_{0}\right|$, since $\left|x^{\prime}-x_{0}\right| \leq\left|x-x_{0}\right|$. Hence $g(\lambda) \leq(1-\lambda) g(0)+\lambda g(1)+C \lambda(1-$ $\lambda)|\xi|^{2}\left|x-x^{\prime}\right|\left|x-x_{0}\right|$, for $0 \leq \lambda \leq 1$. Therefore we get

$$
\begin{aligned}
- & \left\langle D_{x}^{2} \phi\left(x_{0}, Y(\lambda), X_{0}\right)\left(x-x_{0}\right), x-x_{0}\right\rangle \\
= & f(\lambda)+g(\lambda) \\
\leq & (1-\lambda)(f(0)+g(0))+\lambda(f(1)+g(1))+\lambda(1-\lambda)|\xi|^{2}\left(C_{1}\left|x-x^{\prime}\right|\left|x-x_{0}\right|-C_{2}\left|x^{\prime}-x_{0}\right|^{2}\right) \\
\leq & -\left\langle\left((1-\lambda) D_{x}^{2} \phi\left(x_{0}, \bar{Y}, X_{0}\right)+\lambda D_{x}^{2} \phi\left(x_{0}, \hat{Y}, X_{0}\right)\right)\left(x-x_{0}\right), x-x_{0}\right\rangle \\
& \quad+\lambda(1-\lambda)|\xi|^{2}\left(C_{1}^{\prime}\left|x-x^{\prime}\right|^{2}-C_{2}^{\prime}\left|x-x_{0}\right|^{2}\right),
\end{aligned}
$$

since $\left|x^{\prime}-x_{0}\right|^{2}=\left|x-x_{0}\right|^{2}-\left|x-x^{\prime}\right|^{2}$, with $C_{1}, C_{2}$ positive structural constants. This finishes the proof of (3.5).

We now prove (3.3). Let $\lambda^{\prime} \in[0,1]$ to be chosen later, and let $\lambda \in[1 / 4,3 / 4]$. We have

$$
\begin{aligned}
& \min \left\{\phi\left(x, \bar{Y}, X_{0}\right), \phi\left(x, \hat{Y}, X_{0}\right)\right\}-\phi\left(x, Y(\lambda), X_{0}\right) \\
& \leq\left(1-\lambda^{\prime}\right) \phi\left(x, \bar{Y}, X_{0}\right)+\lambda^{\prime} \phi\left(x, \hat{Y}, X_{0}\right)-\phi\left(x, Y(\lambda), X_{0}\right) \\
& =\left\langle\left(\left(1-\lambda^{\prime}\right) D_{x} \phi\left(x_{0}, \bar{Y}, X_{0}\right)+\lambda^{\prime} D_{x} \phi\left(x_{0}, \hat{Y}, X_{0}\right)\right)-D_{x} \phi\left(x_{0}, Y(\lambda), X_{0}\right), x-x_{0}\right\rangle \\
& +\frac{1}{2}\left\langle\left(\left(1-\lambda^{\prime}\right) D_{x}^{2} \phi\left(x_{0}, \bar{Y}, X_{0}\right)+\lambda^{\prime} D_{x}^{2} \phi\left(x_{0}, \hat{Y}, X_{0}\right)-D_{x}^{2} \phi\left(x_{0}, Y(\lambda), X_{0}\right)\right)\left(x-x_{0}\right), x-x_{0}\right\rangle \\
& +\frac{1}{6} \sum_{i, j, k=1}^{n}\left(\left(1-\lambda^{\prime}\right) D_{i, j, k} \phi\left(\tau, \bar{Y}, X_{0}\right)+\lambda^{\prime} D_{i, j, k} \phi\left(\tau, \hat{Y}, X_{0}\right)-D_{i, j, k} \phi\left(\tau, Y(\lambda), X_{0}\right)\right)\left(x_{i}-x_{0_{i}}\right)\left(x_{j}-x_{0_{j}}\right)\left(x_{k}-x_{0_{k}}\right)
\end{aligned}
$$


for some $\tau \in\left[x_{0}, x\right]$. We now use (3.5) in $\lambda^{\prime}$ to get

$$
\begin{aligned}
\leq & \left\langle\left(D \phi\left(x_{0}, Y\left(\lambda^{\prime}\right), X_{0}\right)-D \phi\left(x_{0}, Y(\lambda), X_{0}\right)\right), x-x_{0}\right\rangle \\
+ & \frac{1}{2}\left\langle\left(D_{x}^{2} \phi\left(x_{0}, Y\left(\lambda^{\prime}\right), X_{0}\right)-D_{x}^{2} \phi\left(x_{0}, Y(\lambda), X_{0}\right)\right)\left(x-x_{0}\right), x-x_{0}\right\rangle \\
& -C_{1} \lambda^{\prime}\left(1-\lambda^{\prime}\right)|\xi|^{2}\left|x-x_{0}\right|^{2}+C_{2} \lambda^{\prime}\left(1-\lambda^{\prime}\right)|\xi|^{2}\left|x-x^{\prime}\right|^{2} \\
+ & \frac{1}{6} \sum_{i, j, k=1}^{n}\left(\left(1-\lambda^{\prime}\right) D_{i, j, k} \phi\left(\tau, \bar{Y}, X_{0}\right)+\lambda^{\prime} D_{i, j, k} \phi\left(\tau, \hat{Y}, X_{0}\right)-D_{i, j, k} \phi\left(\tau, Y(\lambda), X_{0}\right)\right)\left(x_{i}-x_{0_{i}}\right)\left(x_{j}-x_{0_{j}}\right)\left(x_{k}-x_{0_{k}}\right)
\end{aligned}
$$

Notice that $\left\langle\left(D \phi\left(x_{0}, Y\left(\lambda^{\prime}\right), X_{0}\right)-D \phi\left(x_{0}, Y(\lambda), X_{0}\right)\right), x-x_{0}\right\rangle=\left(\lambda^{\prime}-\lambda\right)\left\langle\xi, x-x_{0}\right\rangle=$ $\left(\lambda^{\prime}-\lambda\right)\left\langle\xi, x-x^{\prime}\right\rangle$ by orthogonality. Hence we get that

$$
\begin{aligned}
& \min \left\{\phi\left(x, \bar{Y}, X_{0}\right), \phi\left(x, \hat{Y}, X_{0}\right)\right\}-\phi\left(x, Y(\lambda), X_{0}\right) \\
& \leq-C_{1} \lambda(1-\lambda)|\xi|^{2}\left|x-x_{0}\right|^{2}+\left(\lambda^{\prime}-\lambda\right)\left\langle\xi, x-x^{\prime}\right\rangle \\
& +C_{2} \lambda(1-\lambda)|\xi|^{2}\left|x-x^{\prime}\right|^{2}+C_{1}|\xi|^{2}\left|x-x_{0}\right|^{2}\left(\lambda(1-\lambda)-\lambda^{\prime}\left(1-\lambda^{\prime}\right)\right) \\
& +C_{2}|\xi|^{2}\left|x-x^{\prime}\right|^{2}\left(\lambda^{\prime}\left(1-\lambda^{\prime}\right)-\lambda(1-\lambda)\right) \\
& +\left\langle\left(D_{x}^{2} \phi\left(x_{0}, Y\left(\lambda^{\prime}\right), X_{0}\right)-D_{x}^{2} \phi\left(x_{0}, Y(\lambda), X_{0}\right)\right)\left(x-x_{0}\right), x-x_{0}\right\rangle \\
& +\sum_{i, j, k=1}^{n}\left(\left(1-\lambda^{\prime}\right) D_{i, j, k} \phi\left(\tau, \bar{Y}, X_{0}\right)+\lambda^{\prime} D_{i, j, k} \phi\left(\tau, \hat{Y}, X_{0}\right)-D_{i, j, k} \phi\left(\tau, Y(\lambda), X_{0}\right)\right)\left(x_{i}-x_{0_{i}}\right)\left(x_{j}-x_{0_{j}}\right)\left(x_{k}-x_{0_{k}}\right)
\end{aligned}
$$

We will now choose $\lambda^{\prime}$ and estimate each of the terms. We have that

$$
\begin{aligned}
A & :=\left(\lambda^{\prime}-\lambda\right)\left\langle\xi, x-x^{\prime}\right\rangle+C_{2} \lambda(1-\lambda)|\xi|^{2}\left|x-x^{\prime}\right|^{2} \\
& =\left\langle x-x^{\prime},\left(\lambda^{\prime}-\lambda\right) \xi+C_{2} \lambda(1-\lambda)|\xi|^{2}\left(x-x^{\prime}\right)\right\rangle \\
& =\left\langle x-x^{\prime},|\xi|\left(\left(\lambda^{\prime}-\lambda\right) \frac{\xi}{|\xi|}+C_{2} \lambda(1-\lambda)|\xi|\left(x-x^{\prime}\right)\right)\right\rangle .
\end{aligned}
$$

Notice that either $\frac{x-x^{\prime}}{\left|x-x^{\prime}\right|}=\frac{\xi}{|\xi|}$ or $\frac{x-x^{\prime}}{\left|x-x^{\prime}\right|}=-\frac{\xi}{|\xi|}$. If the + sign holds, we choose $\lambda^{\prime}=$ $\lambda-C_{2} \lambda(1-\lambda)|\xi|\left|x-x^{\prime}\right|$, and if the - sign holds, we choose $\lambda^{\prime}=\lambda+C_{2} \lambda(1-\lambda)\left|\xi \| x-x^{\prime}\right|$, so in either case $A=0$. Notice that since $1 / 4 \leq \lambda \leq 3 / 4$, we have from Lemma 2.1 that $\left|\lambda^{\prime}-\lambda\right| \leq \frac{C_{2}}{16}|\xi|\left|x-x^{\prime}\right| \leq \frac{C_{3}}{16}|\bar{Y}-\hat{Y}|\left|x-x_{0}\right| \leq C\left|x-x_{0}\right| \leq \frac{1}{4}$, if $\left|x-x_{0}\right|<1 / 4 C$. Hence $\lambda^{\prime} \in[0,1]$.

We next estimate the remaining terms. We have $C_{1}|\xi|^{2}\left|x-x_{0}\right|^{2}\left(\lambda(1-\lambda)-\lambda^{\prime}(1-\right.$ $\left.\left.\lambda^{\prime}\right)\right) \leq 3 C_{1}|\xi|^{2}\left|x-x_{0}\right|^{2}\left|\lambda^{\prime}-\lambda\right| \leq C|\bar{Y}-\hat{Y}|^{3}\left|x-x_{0}\right|^{3}$, again by Lemma 2.1. Similarly, $C_{2}|\xi|^{2}\left|x-x^{\prime}\right|^{2}\left(\lambda^{\prime}\left(1-\lambda^{\prime}\right)-\lambda(1-\lambda)\right) \leq C|\bar{Y}-\hat{Y}|^{3}\left|x-x_{0}\right|^{3}$.

We also have from the estimates at the end of Subsection 2.4 that

$$
\begin{aligned}
& \left\langle\left(D_{x}^{2} \phi\left(x_{0}, Y\left(\lambda^{\prime}\right), X_{0}\right)-D_{x}^{2} \phi\left(x_{0}, Y(\lambda), X_{0}\right)\right)\left(x-x_{0}\right), x-x_{0}\right\rangle \\
& \leq C\left|Y\left(\lambda^{\prime}\right)-Y(\lambda)\right|\left|x-x_{0}\right|^{2} \leq C\left|v\left(\lambda^{\prime}\right)-v(\lambda)\right|\left|x-x_{0}\right|^{2} \\
& =\left|\lambda^{\prime}-\lambda\right||\bar{v}-\hat{v}|\left|x-x_{0}\right|^{2} \leq C\left|\lambda^{\prime}-\lambda\right||\bar{Y}-\hat{Y}|\left|x-x_{0}\right|^{2} \leq C|\bar{Y}-\hat{Y}|^{2}\left|x-x_{0}\right|^{3} .
\end{aligned}
$$


To estimate the cubic form, let $h(\lambda)=D_{i, j, k} \phi\left(\tau, Y(\lambda), X_{0}\right)$. As in the estimate of $g^{\prime \prime}$ above, we have $\left|h^{\prime \prime}(\lambda)\right| \leq C|\xi|^{2}$. We have

$$
\begin{aligned}
& \left|\left(\left(1-\lambda^{\prime}\right) D_{i, j, k} \phi\left(\tau, \bar{Y}, X_{0}\right)+\lambda^{\prime} D_{i, j, k} \phi\left(\tau, \hat{Y}, X_{0}\right)-D_{i, j, k} \phi\left(\tau, Y(\lambda), X_{0}\right)\right)\left(x_{i}-x_{0_{i}}\right)\left(x_{j}-x_{0_{j}}\right)\left(x_{k}-x_{0_{k}}\right)\right| \\
& \leq C\left|x-x_{0}\right|^{3}\left|\left(1-\lambda^{\prime}\right) h(0)+\lambda^{\prime} h(1)-h(\lambda)\right| \\
& \leq C\left|x-x_{0}\right|^{3}\left|\left(1-\lambda^{\prime}\right) h(0)+\lambda^{\prime} h(1)-h\left(\lambda^{\prime}\right)\right|+C\left|x-x_{0}\right|^{3}\left|h\left(\lambda^{\prime}\right)-h(\lambda)\right| \\
& \leq C\left|x-x_{0}\right|^{3}|\xi|^{2}+C\left|x-x_{0}\right|^{3}\left|\lambda^{\prime}-\lambda\right||\xi| \\
& \leq C\left|x-x_{0}\right|^{3}|\bar{Y}-\hat{Y}|^{2}+C\left|x-x_{0}\right|^{4}|\bar{Y}-\hat{Y}|^{2} .
\end{aligned}
$$

Combining all these estimates we obtain

$$
\begin{aligned}
& \min \left\{\phi\left(x, \bar{Y}, X_{0}\right), \phi\left(x, \hat{Y}, X_{0}\right)\right\}-\phi\left(x, Y(\lambda), X_{0}\right) \\
& \leq-C_{1}^{\prime}|\bar{Y}-\hat{Y}|^{2}\left|x-x_{0}\right|^{2}+C\left(|\bar{Y}-\hat{Y}|^{3}\left|x-x_{0}\right|^{3}+|\bar{Y}-\hat{Y}|^{2}\left|x-x_{0}\right|^{3}+|\bar{Y}-\hat{Y}|^{2}\left|x-x_{0}\right|^{4}\right),
\end{aligned}
$$

where $C_{1}^{\prime}$ and $C$ are structural constants. To obtain our desired estimate we write

$$
\begin{aligned}
& C\left(|\bar{Y}-\hat{Y}|^{3}\left|x-x_{0}\right|^{3}+|\bar{Y}-\hat{Y}|^{2}\left|x-x_{0}\right|^{3}+|\bar{Y}-\hat{Y}|^{2}\left|x-x_{0}\right|^{4}\right) \\
& =C\left|x-x_{0}\right|^{2}|\bar{Y}-\hat{Y}|^{2}\left(|\bar{Y}-\hat{Y}|\left|x-x_{0}\right|+\left|x-x_{0}\right|+\left|x-x_{0}\right|^{2}\right) \\
& \leq \frac{C_{1}^{\prime}}{2}\left|x-x_{0}\right|^{2}|\bar{Y}-\hat{Y}|^{2},
\end{aligned}
$$

provided we choose $\left|x-x_{0}\right| \leq C_{2}^{\prime}$, with $C_{2}^{\prime}$ sufficiently small depending only on the structure. Taking $\sigma=\min \left\{1 / 4 C, C_{2}^{\prime}\right\}$, the first part of the lemma follows.

We finally show that (3.3) implies (3.2). Fix $X_{0} \in C_{\Omega}, Y_{0} \in \Sigma$ and let $\xi$ and $\eta$ be perpendicular vectors in $R^{n}$. Set $v_{0}=D \phi\left(x_{0}, Y_{0}, X_{0}\right)$ and hence we can write $Y_{0}=X_{0}+s_{X_{0}}\left(\Lambda\left(v_{0}\right)\right) \Lambda\left(v_{0}\right)$. Let $Y_{\epsilon} \in \Sigma$ be given by $\left.Y_{\epsilon}=X_{0}+s\left(\Lambda\left(v_{0}+\epsilon \xi\right)\right) \Lambda\left(v_{0}+\epsilon \xi\right)\right)$ and notice that $Y_{0} \in\left[Y_{-\epsilon}, Y_{\epsilon}\right]_{X_{0}}$ for the value $\lambda=\frac{1}{2}$ and for all $\epsilon>0$. Therefore from (3.3) we have $\phi\left(x, Y_{0}, X_{0}\right) \geq \min \left\{\phi\left(x, Y_{-\epsilon}, X_{0}\right), \phi\left(x, Y_{\epsilon}, X_{0}\right)\right\}+C_{1} \epsilon^{2}|\xi|^{2}\left|x-x_{0}\right|^{2}$ for $\left|x-x_{0}\right| \leq C_{2}$.

Let $S_{\epsilon}=\left\{x \in \Omega: \phi\left(x, Y_{-\epsilon}, X_{0}\right)=\phi\left(x, Y_{\epsilon}, X_{0}\right)\right\}$. Notice that $D \phi\left(x_{0}, Y_{\epsilon}, X_{0}\right)-$ $D \phi\left(x_{0}, Y_{-\epsilon}, X_{0}\right)=2 \epsilon \xi$ is a normal vector to $S_{\epsilon}$ at $x_{0}$.

Let $\gamma$ be a curve contained in $S_{\epsilon}$ such that $\gamma(0)=x_{0}$ and $\gamma^{\prime}(0)=\eta$. We then have that $\left.\phi\left(\gamma(t), Y_{0}, X_{0}\right) \geq \frac{1}{2} \phi\left(\gamma(t), Y_{-\epsilon}, X_{0}\right)+\frac{1}{2} \phi\left(\gamma(t), Y_{\epsilon}, X_{0}\right)\right\}+C_{1} \epsilon^{2}|\xi|^{2}\left|\gamma(t)-x_{0}\right|^{2}$ for all $|t|$ small enough.

Let $\left.g(t)=\phi\left(\gamma(t), Y_{0}, X_{0}\right)-\frac{1}{2} \phi\left(\gamma(t), Y_{-\epsilon}, X_{0}\right)-\frac{1}{2} \phi\left(\gamma(t), Y_{\epsilon}, X_{0}\right)\right\}-C_{1} \epsilon^{2}|\xi|^{2}\left|\gamma(t)-x_{0}\right|^{2}$.

We have $g^{\prime}(0)=0$ and $g^{\prime \prime}(0) \geq 0$ and that is

$$
\left\langle\left(D_{x}^{2} \phi\left(x_{0}, Y_{0}, X_{0}\right)-\frac{1}{2} D_{x}^{2} \phi\left(x_{0}, Y_{-\epsilon}, X_{0}\right)-\frac{1}{2} D_{x}^{2} \phi\left(x_{0}, Y_{\epsilon}, X_{0}\right)-2 C_{1} \epsilon^{2}|\xi|^{2} I d\right) \eta, \eta\right\rangle \geq 0
$$

and this inequality holds for all $\epsilon$ small enough. Letting $h(\epsilon)=\left\langle\left(D_{x}^{2} \phi\left(x_{0}, Y_{\epsilon}, X_{0}\right)\right) \eta, \eta\right\rangle$, we get that $h(0)-\frac{1}{2} h(-\epsilon)-\frac{1}{2} h(\epsilon) \geq 2 C_{1} \epsilon^{2}|\xi|^{2}|\eta|^{2}$, we get that $h^{\prime \prime}(0) \leq-4 C_{1}|\xi|^{2}|\eta|^{2}$. Therefore we obtain that $\left.\frac{d^{2}}{d \epsilon^{2}}\left\langle D_{x}^{2} \phi\left(x_{0}, Y_{\epsilon}, X_{0}\right) \eta, \eta\right\rangle\right|_{\epsilon=0} \leq-C|\xi|^{2}|\eta|^{2}$. 
Remark 3.3. The local version of (3.2) can be stated as follows: The target $\Sigma$ is regular from $X_{0} \in C_{\Omega}$ if there exists a neighborhood $U_{X_{0}}$ and a constant $C$ depending on $X_{0}$ such that for all $Y_{0} \in \Sigma$ and for all $Z \in U_{X_{0}}$ and for all vectors $\xi$ and $\eta$ such that $\xi \perp \eta$ we have

$$
\left.\frac{d^{2}}{d \epsilon^{2}}\left\langle D_{x}^{2} \phi\left(z, Y_{\epsilon}, Z\right) \eta, \eta\right\rangle\right|_{\epsilon=0} \leq-C|\xi|^{2}|\eta|^{2}
$$

where $Y_{\epsilon}=Z+s(\Lambda(v+\epsilon \xi)) \Lambda(v+\epsilon \xi)$ and $v=D \phi\left(z, Y_{0}, Z\right)$.

Following the proof of Theorem 3.2 , one can show that $(3.6)$ is equivalent to (3.1).

\section{Definition of REFRACTOR AND MAIN RESUlts}

4.1. Refractors. Let $u: \Omega \rightarrow[0, M]$, and assume that the convex hull of $\Sigma \subset \mathcal{T}$, and $\Omega$ is connected. Given $x_{0} \in \Omega$, set $X_{0}=\left(x_{0}, u\left(x_{0}\right)\right)$. We define

$$
F_{u}\left(x_{0}\right)=\left\{Y \in \Sigma: u(x) \leq \phi\left(x, Y, X_{0}\right) \text { for all } x \in \Omega\right\} .
$$

The function $u$ is a parallel refractor if $F_{u}\left(x_{0}\right) \neq \emptyset$ for all $x_{0} \in \Omega$.

We notice that, from the estimates of the derivatives $\partial_{x_{i}} \phi$ from Subsection 2.4. any refractor is a Lipschitz function in $\Omega$ with a Lipschitz constant depending only on $\delta$ in 2.6 .

Lemma 4.1. If $u$ is a refractor, then for each $\bar{x}, \hat{x} \in \Omega$ and each $s \in[0,1]$ we have $u((1-s) \bar{x}+s \hat{x}) \geq(1-s) u(\bar{x})+s u(\hat{x})-C|\bar{x}-\hat{x}|^{2} s(1-s)$, with $C$ is a structural constant.

Proof. We use the fact from Subsection 2.4 that $\left|D_{x_{i} x_{j}} \phi(x, Y, \bar{X})\right| \leq C$ for any $x \in \Omega$, $\bar{X} \in C_{\Omega}$ and $Y \in \mathcal{T}$.

Given $\tilde{x} \in \Omega$, let $\tilde{Y} \in F_{u}(\tilde{x})$ and set $\tilde{X}=(\tilde{x}, u(\tilde{x}))$. We have $u(x) \leq \phi(x, \tilde{Y}, \tilde{X}) \leq$ $\phi(\tilde{x}, \tilde{Y}, \tilde{X})+\langle D \phi(\tilde{x}, \tilde{Y}, \tilde{X}), x-\tilde{x}\rangle+C|x-\tilde{x}|^{2}=u(\tilde{x})+\langle p, x-\tilde{x}\rangle+C|x-\tilde{x}|^{2}$ for all $x \in \Omega$. Next, given $s \in[0,1]$, let $x_{s}=(1-s) \bar{x}+s \hat{x}, Y \in F_{u}\left(x_{s}\right)$, and $X_{s}=\left(x_{s}, u\left(x_{s}\right)\right)$. Applying the previous inequality $\tilde{x} \leadsto x_{s}, \tilde{Y} \leadsto Y$, and $p \leadsto D \phi\left(x_{s}, Y, X_{s}\right)$, we get $u(x) \leq u\left(x_{s}\right)+\left\langle p, x-x_{s}\right\rangle+C\left|x-x_{s}\right|^{2}$ for all $x \in \Omega$.

Let $\psi(t)=u\left(x_{t}\right)$. We have $\psi(t) \leq u\left(x_{s}\right)+\left\langle p, x_{t}-x_{s}\right\rangle+C\left|x_{t}-x_{s}\right|^{2}=\psi(s)+(t-$ $s)\langle p, \hat{x}-\bar{x}\rangle+C(t-s)^{2}|\bar{x}-\hat{x}|^{2}$. Therefore $\psi(0) \leq \psi(s)-s\langle p, \hat{x}-\bar{x}\rangle+C s^{2}|\bar{x}-\hat{x}|^{2}$ and $\psi(1) \leq \psi(s)+(1-s)\langle p, \hat{x}-\bar{x}\rangle+C(1-s)^{2}|\bar{x}-\hat{x}|^{2}$. Proceeding as in Theorem 3.2. we obtain $(1-s) \psi(0)+s \psi(1) \leq \psi(s)+C s(1-s)|\bar{x}-\hat{x}|^{2}$ and the lemma is proved.

We will next prove our main lemma.

Lemma 4.2. Suppose $u$ is a parallel refractor and the target $\Sigma$ is regular from $X^{\star}=$ $\left(x^{\star}, u\left(x^{\star}\right)\right)$ in the sense of Definition 3.1. There exists constants $\delta, C_{1}$ and $C_{2}$ depending on $X^{\star}$ such that if $\bar{x}, \hat{x} \in B_{\delta}\left(x^{\star}\right)$ and $\bar{Y} \in F_{u}(\bar{x}), \hat{Y} \in F_{u}(\hat{x})$ and $|\bar{Y}-\hat{Y}| \geq|\bar{x}-\hat{x}|$; then there exists $x_{0} \in \overline{\bar{x}} \hat{x}$ such that if $X_{0}^{\star}=\left(x_{0}, u\left(x_{0}\right)\right)$, then

$$
u(x)-\phi\left(x, Y, X_{0}^{\star}\right) \leq C|\bar{Y}-\hat{Y}||\bar{x}-\hat{x}|+C|Y(\lambda)-Y|\left|x-x_{0}\right|-C_{1}|\bar{Y}-\hat{Y}|^{2}\left|x-x_{0}\right|^{2}
$$

for all $Y(\lambda) \in[\bar{Y}, \hat{Y}]_{X_{0}^{\star}}, 1 / 4 \leq \lambda \leq 3 / 4, Y \in \Sigma$ and for all $x \in \Omega \cap B_{C_{2}}\left(x_{0}\right)$. 
We remark that $C$ is a structural constant, depending only on the bounds for the derivatives of $\phi$.

Proof. Since $\Sigma$ is regular from $X^{\star}$, there exists a neighborhood $U_{X^{\star}}$ of $X^{\star}$ such that (3.1) holds for all $\bar{Y}, \hat{Y} \in \Sigma$ and all $Z \in U_{X^{\star}}$. Since parallel refractors are uniformly Lipschitz in $\Omega$, there exists $\delta>0$ such that $(x, u(x)) \in U_{X^{\star}}$ for all $x \in B_{\delta}\left(x^{\star}\right)$.

If $\bar{Y} \in F_{u}(\bar{x})$ and $\hat{Y} \in F_{u}(\hat{x})$, it follows that $u(x) \leq \min \{\phi(x, \bar{Y}, \bar{X}), \phi(x, \hat{Y}, \hat{X})\}$ for all $x \in \Omega$ with $\bar{X}=(\bar{x}, u(\bar{x})), \hat{X}=(\hat{x}, u(\hat{x}))$. By continuity there exists $x_{0} \in[\bar{x}, \hat{x}]$ such that $\phi\left(x_{0}, \bar{Y}, \bar{X}\right)=\phi\left(x_{0}, \hat{Y}, \hat{X}\right)$. Indeed, setting $h(x)=\phi(x, \bar{Y}, \bar{X})-\phi(x, \hat{Y}, \hat{X})$, we have that $h(\bar{x})=u(\bar{x})-\phi(\bar{x}, \hat{Y}, \hat{X}) \leq 0$ and $h(\hat{x})=\phi(\hat{x}, \bar{Y}, \bar{X})-u(\hat{x}) \geq 0$.

Now set $x_{0_{n+1}}=\phi\left(x_{0}, \bar{Y}, \bar{X}\right)=\phi\left(x_{0}, \hat{Y}, \hat{X}\right), X_{0}=\left(x_{0}, x_{0_{n+1}}\right)$, and recall we have set $X_{0}^{\star}=\left(x_{0}, u\left(x_{0}\right)\right) \in U_{X^{\star}}$ and $u\left(x_{0}\right) \leq x_{0_{n+1}}$. By definition $\phi(x, \hat{Y}, \hat{X})=\phi\left(x, \hat{Y}, X_{0}\right)$ and $\phi(x, \bar{Y}, \bar{X})=\phi\left(x, \bar{Y}, X_{0}\right)$ for all $x \in \Omega$. Hence we can write

$$
u(x) \leq \min \left\{\phi\left(x, \bar{Y}, X_{0}\right), \phi\left(x, \hat{Y}, X_{0}\right)\right\}=\min \left\{\phi\left(x, \bar{Y}, X_{0}^{\star}\right), \phi\left(x, \hat{Y}, X_{0}^{\star}\right)\right\}+E .
$$

We now estimate $E$. First, notice that by Lemma (2.2), we have $0 \leq E \leq C\left(x_{0_{n+1}}-\right.$ $\left.u\left(x_{0}\right)\right)$. We claim that

$$
x_{0_{n+1}}-u\left(x_{0}\right) \leq C|\bar{Y}-\hat{Y} \| \bar{x}-\hat{x}| .
$$

We can write $x_{0}=(1-s) \bar{x}+s \hat{x}$ for some $0<s<1$. Then, by Lemma (4.1), we have $u\left(x_{0}\right) \geq(1-s) u(\bar{x})+s u(\hat{x})-C|\bar{x}-\hat{x}|^{2} s(1-s)$. Since $u(\bar{x})=\phi\left(\bar{x}, \bar{Y}, X_{0}\right)$ and $u(\hat{x})=\phi\left(\hat{x}, \hat{Y}, X_{0}\right)$, we get

$$
x_{0_{n+1}}-u\left(x_{0}\right) \leq x_{0_{n+1}}-\left((1-s) \phi\left(\bar{x}, \bar{Y}, X_{0}\right)+s \phi\left(\hat{x}, \hat{Y}, X_{0}\right)\right)+C|\bar{x}-\hat{x}|^{2} s(1-s) .
$$

On the other hand, $\left.\left.\phi\left(\hat{x}, \hat{Y}, X_{0}\right)\right) \geq \phi\left(x_{0}, \hat{Y}, X_{0}\right)\right)+\left\langle D \phi\left(x_{0}, \hat{Y}, X_{0}\right), \hat{x}-x_{0}\right\rangle$, and $\left.\phi\left(\bar{x}, \bar{Y}, X_{0}\right)\right) \geq$ $\left.\phi\left(x_{0}, \bar{Y}, X_{0}\right)\right)+\left\langle D \phi\left(x_{0}, \hat{Y}, X_{0}\right), \bar{x}-x_{0}\right\rangle$ by convexity. Using that $\bar{x}-x_{0}=s(\bar{x}-\hat{x})$, and $\hat{x}-x_{0}=-(1-s)(\bar{x}-\hat{x})$ we obtain

$$
\begin{aligned}
\phi\left(\hat{x}, \hat{Y}, X_{0}\right) & \geq x_{0_{n+1}}-(1-s)\left\langle D \phi\left(x_{0}, \hat{Y}, X_{0}\right), \bar{x}-\hat{x}\right\rangle, \text { and } \\
\left.\phi\left(\bar{x}, \bar{Y}, X_{0}\right)\right) & \geq x_{0_{n+1}}+s\left\langle D \phi\left(x_{0}, \bar{Y}, X_{0}\right), \bar{x}-\hat{x}\right\rangle .
\end{aligned}
$$

It follows that

$$
\begin{aligned}
& \left.(1-s) \phi\left(\bar{x}, \bar{Y}, X_{0}\right)\right)+s \phi\left(\hat{x}, \hat{Y}, X_{0}\right) \\
& \geq x_{0_{n+1}}+(1-s) s\left\langle\left(D \phi\left(x_{0}, \bar{Y}, X_{0}\right)-D \phi\left(x_{0}, \hat{Y}, X_{0}\right)\right), \bar{x}-\hat{x}\right\rangle \\
& \geq x_{0_{n+1}}-s(1-s)\left|\left\langle\left(D \phi\left(x_{0}, \bar{Y}, X_{0}\right)-D \phi\left(x_{0}, \hat{Y}, X_{0}\right)\right), \bar{x}-\hat{x}\right\rangle\right| \\
& \geq x_{0_{n+1}}-s(1-s)\left|D \phi\left(x_{0}, \bar{Y}, X_{0}\right)-D \phi\left(x_{0}, \hat{Y}, X_{0}\right)\right||\bar{x}-\hat{x}| \\
& \geq x_{0_{n+1}}-s(1-s) C|\bar{Y}-\hat{Y}||\bar{x}-\hat{x}|,
\end{aligned}
$$

from the estimates for the derivatives of $\phi$ in $Y$. Therefore, inserting the last estimate in $(4.4)$ we obtain $x_{0_{n+1}}-u\left(x_{0}\right) \leq C s(1-s)\left(|\bar{Y}-\hat{Y}||\bar{x}-\hat{x}|+|\bar{x}-\hat{x}|^{2}\right) \leq C \mid \bar{Y}-$ 
$\hat{Y}|| \bar{x}-\hat{x} \mid$, where in the last inequality we have used that $s \in[0,1]$ and $|\bar{x}-\hat{x}| \leq|\bar{Y}-\hat{Y}|$ by assumption. We then obtain claim (4.3).

This yields

$$
u(x) \leq \min \left\{\phi\left(x, \bar{Y}, X_{0}^{\star}\right), \phi\left(x, \hat{Y}, X_{0}^{\star}\right)\right\}+C|\bar{Y}-\hat{Y} \| \bar{x}-\hat{x}| .
$$

If $\bar{x}, \hat{x} \in B_{\delta}\left(x^{\star}\right)$, then $X_{0}^{*} \in U_{X^{\star}}$. So if $Y(\lambda) \in[\bar{Y}, \hat{Y}]_{X_{0}^{\star}}$, then we can apply (3.1) to get $\min \left\{\phi\left(x, \bar{Y}, X_{0}^{\star}\right), \phi\left(x, \hat{Y}, X_{0}^{\star}\right)\right\} \leq \phi\left(x, Y(\lambda), X_{0}^{\star}\right)-C_{1}|\bar{Y}-\hat{Y}|^{2}\left|x-x_{0}\right|^{2}$, for $x \in B_{C_{2}}\left(x_{0}\right) \cap \Omega$ where the constants $C_{1}, C_{2}$ depend on $X^{\star}$. And so

$$
u(x) \leq \phi\left(x, Y(\lambda), X_{0}^{\star}\right)+C|\bar{Y}-\hat{Y}||\bar{x}-\hat{x}|-C_{1}|\bar{Y}-\hat{Y}|^{2}\left|x-x_{0}\right|^{2} .
$$

Finally, from Lemma (2.3), we have that $\left|\phi\left(x, Y(\lambda), X_{0}^{\star}\right)-\phi\left(x, Y, X_{0}^{\star}\right)\right| \leq C \mid Y(\lambda)-$ $Y|| x-x_{0} \mid$, and consequently $u(x)-\phi\left(x, Y, X_{0}^{\star}\right) \leq C|\bar{Y}-\hat{Y}||\bar{x}-\hat{x}|+C|Y(\lambda)-Y|\left|x-x_{0}\right|-$ $C_{1}|\bar{Y}-\hat{Y}|^{2}\left|x-x_{0}\right|^{2}$, where the constant $C$ is structural. This completes the proof of the lemma.

4.2. Local and global refractors. Suppose that $u$ is a parallel refractor in $\Omega$. In general, a local supporting ellipsoid might not support the refractor in all of $\Omega$. For example, rotating the Figure 3 around the $z$-axis, we obtain a refractor in $3 \mathrm{~d}$ that has a local supporting ellipsoid that is not global. In this case, the target is composed of the circle $x^{2}+y^{2}=(.03)^{2}$ with $z=0$, and the point $(0,0,10)$.

The purpose of this subsection is to see that under condition $(4.5)$ below, a local supporting ellipsoid is also global. This will be used later in the proof of Theorem 4.5 .

The target $\Sigma$ satisfies the condition AW from $X_{0} \in C_{\Omega}$ if for all $Y_{0} \in \Sigma$, and for all vectors $\xi$ and $\eta$ such that $\xi \perp \eta$ we have

$$
\left.\frac{d^{2}}{d \epsilon^{2}}\left\langle D_{x}^{2} \phi\left(x_{0}, Y_{\epsilon}, X_{0}\right) \eta, \eta\right\rangle\right|_{\epsilon=0} \leq 0
$$

where $Y_{\epsilon}=X_{0}+s(\Lambda(v+\epsilon \xi)) \Lambda(v+\epsilon \xi)$ and $v=D \phi\left(x_{0}, Y_{0}, X_{0}\right)$. Clearly (3.6) implies (4.5). We will show in Proposition 4.3 that (4.5) implies that for all $\bar{Y}, \hat{Y} \in \Sigma$ we have

$$
\phi\left(x, Y_{X_{0}}(\lambda), X_{0}\right) \geq \min \left\{\phi\left(x, \bar{Y}, X_{0}\right), \phi\left(x, \hat{Y}, X_{0}\right)\right\}
$$

for all $x \in \Omega$, and $0 \leq \lambda \leq 1$, with $Y_{X_{0}}(\lambda)=X_{0}+s_{Z}(\Lambda(v(\lambda))) \Lambda(v(\lambda))$. Here $\bar{v}=D_{x} \phi\left(x_{0}, \bar{Y}, X_{0}\right), \hat{v}=D_{x} \phi\left(x_{0}, \hat{Y}, X_{0}\right)$, and $v(\lambda)=(1-\lambda) \bar{v}+\lambda \hat{v}$.

Let $H(v, X):=s_{X}(\Lambda(v)) Q(v)$, see (2.1) and Subsection 2.5. Condition (4.5) means that for all $\eta, \xi \in \mathbb{R}^{n}$, with $\eta \perp \xi$, we have

$$
D_{v_{l}, v_{k}}\left(D_{x_{i}, x_{j}} \phi(x, Y(v), X) \eta_{i} \eta_{j} \xi_{k} \xi_{l} \leq 0\right.
$$

with $X=X_{0}=\left(x_{0}, x_{n+1}^{0}\right)$. We are going to rewrite this condition in terms of the function $H$. We will show that this is equivalent to

$$
\left\langle D_{v}^{2}(1 / H(v, X)) \xi, \xi\right\rangle \leq 0, \quad \text { for all } \xi \in R^{n} .
$$




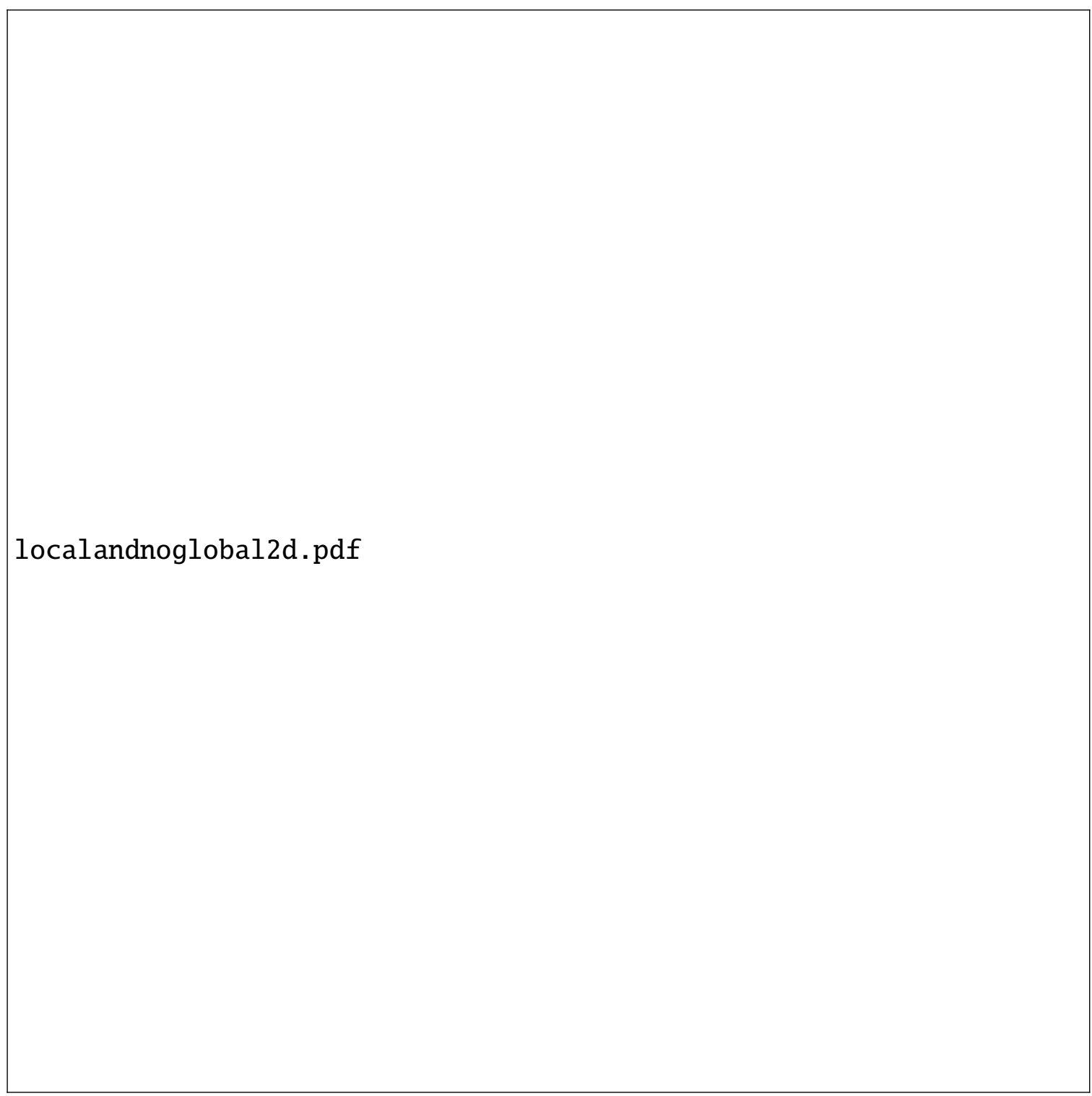

Figure 3. The refractor $R$ is composed of the minimum of the ellipsoids given by $\phi\left(x, Y_{1}, P\right)$, and $\phi\left(x, Y_{2}, P\right)$, with $Y_{1}=(.03,5), Y_{2}=$ $(-.03,5)$ and $P=(0,4.70456)$. The ellipsoid given by $\phi\left(x, Y_{3}, P\right)$ with $Y_{3}=(0,10)$, supports $R$ at $P$ locally but not globally; $\kappa=2 / 3$.

Recall that from Subsection 2.4 we have that $D_{x_{i}} \phi(x, Y, X)=\frac{x_{i}-y_{i}}{y_{n+1}-x_{n+1}-k|X-Y|^{\prime}}$, where $x_{n+1}=\phi(x, Y, X)$. Let us set

$$
J(Y, X, \eta):=\left\langle D_{x}^{2} \phi(x, Y, X) \eta, \eta\right\rangle=\sum_{i, j=1}^{n} D_{x_{i}, x_{j}} \phi(x, Y, X) \eta_{i} \eta_{j} .
$$


From (2.10) and (2.9) we have

$D_{x_{i}, x_{j}} \phi(x, Y, X)=\delta_{i j}\left(y_{n+1}-x_{n+1}-\kappa|X-Y|\right)^{-1}+\left(1-\kappa^{2}\right)\left(y_{n+1}-x_{n+1}-\kappa|X-Y|\right)^{-3}\left(x_{i}-y_{i}\right)\left(x_{j}-y_{j}\right)$.

Therefore, for $|\eta|=1$ we get

$J(Y, X, \eta)=\left(y_{n+1}-x_{n+1}-\kappa|X-Y|\right)^{-1}+\left(1-\kappa^{2}\right)\left(y_{n+1}-x_{n+1}-\kappa|X-Y|\right)^{-3}\langle x-y, \eta\rangle^{2}$.

From (2.1), $\Lambda(v)=(-Q(v) v, Q(v)+\kappa)$. Then

$$
Y_{\epsilon}-X=s_{X}(\Lambda(v))(-Q(v) v, Q(v)+\kappa) \text {. }
$$

Therefore, $J\left(Y_{\epsilon}, X, \eta\right)=\frac{1+\left(1-\kappa^{2}\right)\langle v, \eta\rangle^{2}}{s_{X}(\Lambda(v)) Q(v)}:=F(v, X, \eta)$. We will show that $\frac{d^{2}}{d \epsilon^{2}} F(v+$ $\epsilon \xi, X, \eta)\left.\right|_{\epsilon=0} \leq 0$. Calculating the second derivative with respect to $\epsilon$, we have that the last inequality is equivalent to

$$
\left.\frac{d^{2}}{d \epsilon^{2}} F(v+\epsilon \xi, X, \eta)\right|_{\epsilon=0}=\sum_{k, \ell=1}^{n} D_{v_{k}, v_{\ell}} F(v, X, \eta) \xi_{k} \xi_{\ell} \leq 0
$$

for all vectors $\xi \perp \eta$.

If we set $G=1 / H$, then $F(v, X, \eta)=G(v, X)\left(1+\left(1-\kappa^{2}\right)\langle v, \eta\rangle^{2}\right)$. A calculation gives that

$$
\begin{aligned}
\sum_{k, \ell=1}^{n} D_{v_{k}, v_{\ell}} F(v, X, \eta) \xi_{k} \xi_{\ell}= & \left(1+\left(1-\kappa^{2}\right)\langle v, \eta\rangle^{2}\right)\left\langle D^{2} G(v, X) \xi, \xi\right\rangle \\
& +4\left(1-\kappa^{2}\right)\langle v, \eta\rangle\langle\xi, \eta\rangle\langle D G(v, X), \xi\rangle+2\left(1-\kappa^{2}\right)\langle\eta, \xi\rangle^{2} G(v, X) \\
= & \left(1+\left(1-\kappa^{2}\right)\langle v, \eta\rangle^{2}\right)\left\langle D^{2} G(v, X) \xi, \xi\right\rangle
\end{aligned}
$$

since $\xi \perp \eta$.

Therefore we have shown that condition $(4.5)$ is equivalent to $(4.8)$.

For simplicity in the notation we assume that $X_{0}=0$ and consider the solid ellipsoids

$$
E(Y)=\left\{X \in \mathbb{R}^{n+1}: c(X, Y) \leq c(0, Y)\right\},
$$

where $c(X, Y)$ is defined by (2.3). From Subsection 2.5, we recall that the target $\Sigma$ is given parametrically from the origin by

$$
Y=s(\Lambda(v)) \Lambda(v)
$$

where $\Lambda(v)=(-Q(v) v, Q(v)+\kappa)$ and $|\Lambda(v)|=1$, with $Q(v)$ given in (2.1).

Proposition 4.3. Suppose (4.5) holds from $X_{0}$ (assumed for simplicity 0). Let $\bar{Y}, \hat{Y} \in \Sigma$ be given by $\bar{Y}=s(\Lambda(\bar{v})) \Lambda(\bar{v})$ and $\hat{Y}=s(\Lambda(\hat{v})) \Lambda(\hat{v})$, and let $v_{\lambda}=(1-\lambda) \bar{v}+\lambda \hat{v}$ for some $\lambda \in(0,1)$, and let $Y_{\lambda}=s\left(\Lambda\left(v_{\lambda}\right)\right) \Lambda\left(v_{\lambda}\right)$. Set $H(v, 0)=H(v)$. If

$$
\frac{1}{H\left(v_{\lambda}\right)} \geq(1-\lambda) \frac{1}{H(\bar{v})}+\lambda \frac{1}{H(\hat{v})},
$$


then

$$
E\left(Y_{\lambda}\right) \subseteq E(\bar{Y}) \cup E(\hat{Y})
$$

and in particular, $\phi\left(x, Y_{\lambda}, X_{0}\right) \geq \min \left\{\phi\left(x, \bar{Y}, X_{0}\right), \phi\left(x, \hat{Y}, X_{0}\right)\right\}$ for all $x$ in their common domain (in particular for $x \in \Omega$ ).

Proof. We first notice that the set $b d r y E(\hat{Y}) \cap b d r y E\left(Y_{\lambda}\right)$ is contained on a hyperplane $\hat{T}$. Indeed, for $X \in \operatorname{bdry} E(\hat{Y}) \cap b \operatorname{dry} E\left(Y_{\lambda}\right)$ we have that $c(X, \hat{Y})=c(0, \hat{Y})$, so $\mid X-$ $\left.\hat{Y}\right|^{2}=\left(|\hat{Y}|-\kappa x_{n+1}\right)^{2}$ which gives that $|X|^{2}-2\langle X, \hat{Y}\rangle+2 \kappa x_{n+1}|\hat{Y}|-\kappa^{2} x_{n+1}^{2}=0$. Also $c\left(X, Y_{\lambda}\right)=c\left(0, Y_{\lambda}\right)$, and so $|X|^{2}-2\left\langle X, Y_{\lambda}\right\rangle+2 \kappa x_{n+1}\left|Y_{\lambda}\right|-\kappa^{2} x_{n+1}^{2}=0$. Subtracting these identities yields $\langle X, \hat{\eta}\rangle=0$ where $\hat{\eta}=\hat{Y}-\kappa|\hat{Y}| e_{n+1}-\left(Y_{\lambda}-\kappa\left|Y_{\lambda}\right| e_{n+1}\right)$, and so $\operatorname{bdry} E(\hat{Y}) \cap \operatorname{bdry} E\left(Y_{\lambda}\right) \subseteq \hat{T}:=\{X:\langle X, \hat{\eta}\rangle=0\}$. In the same way, $\operatorname{bdry} E(\bar{Y}) \cap$ $\operatorname{bdry} E\left(Y_{\lambda}\right) \subseteq \bar{T}$, where $\bar{T}=\{X:\langle X, \bar{\eta}\rangle=0\}$ and $\bar{\eta}=\bar{Y}-\kappa|\bar{Y}| e_{n+1}-\left(Y_{\lambda}-\kappa\left|Y_{\lambda}\right| e_{n+1}\right)$.

From (2.1) and the definition of $H$, we can write $\hat{\eta}=\left(H\left(v_{\lambda}\right) v_{\lambda}-H(\hat{v}) \hat{v}, H(\hat{v})-H\left(v_{\lambda}\right)\right)$ and $\bar{\eta}=\left(H\left(v_{\lambda}\right) v_{\lambda}-H(\bar{v}) \bar{v}, H(\bar{v})-H\left(v_{\lambda}\right)\right)$.

The proposition will follow from the following claims:

Claim 1: If $\langle X, \hat{\eta}\rangle \geq 0$ and $X \in E\left(Y_{\lambda}\right)$, then $X \in E(\hat{Y})$.

Claim 2: If $\langle X, \bar{\eta}\rangle \geq 0$ and $X \in E\left(Y_{\lambda}\right)$, then $X \in E(\bar{Y})$.

Claim 3: If $\langle X, \hat{\eta}\rangle<0$ and $\langle X, \bar{\eta}\rangle<0$, then $X \notin E\left(Y_{\lambda}\right)$.

Only the proof of the third claim uses condition $A W$, i.e., 4.10).

We prove Claim 1. Let $\langle X, \hat{\eta}\rangle \geq 0$ and $X \in E\left(Y_{\lambda}\right)$. Since $X \in E\left(Y_{\lambda}\right)$, we have $|X|^{2}-2\left\langle X, Y_{\lambda}\right\rangle+2 \kappa x_{n+1}\left|Y_{\lambda}\right|-\kappa^{2} x_{n+1}^{2} \leq 0$; and since $\langle X, \hat{\eta}\rangle \geq 0$ we have $\left\langle X, Y_{\lambda}-\hat{Y}\right\rangle-$ $\kappa x_{n+1}\left(\left|Y_{\lambda}\right|-|\hat{Y}|\right) \leq 0$. Therefore,

$$
\begin{aligned}
& |X|^{2}-2\langle X, \hat{Y}\rangle+2 \kappa x_{n+1}|\hat{Y}|-\kappa^{2} x_{n+1}^{2} \\
& =|X|^{2}-2\left\langle X, Y_{\lambda}\right\rangle+2 \kappa x_{n+1}\left|Y_{\lambda}\right|-\kappa^{2} x_{n+1}^{2}+2\left\langle X, Y_{\lambda}-\hat{Y}\right\rangle-2 \kappa x_{n+1}\left(\left|Y_{\lambda}\right|-|\hat{Y}|\right) \leq 0 .
\end{aligned}
$$

It follows that $|X-\hat{Y}|^{2} \leq\left(|\hat{Y}|-\kappa x_{n+1}\right)^{2}$. In this inequality, writing $X=\left(x, x_{n+1}\right), \hat{Y}=$ $\left(\hat{y}, \hat{y}_{n+1}\right)$, and completing the squares we obtain, since $0<\kappa<1$, that $|\hat{Y}|-\kappa x_{n+1} \geq 0$ and hence $|X-\hat{Y}| \leq|\hat{Y}|-\kappa x_{n+1}$, which means $X \in E(\hat{Y})$.

The proof of Claim 2 is exactly the same.

We now prove Claim 3.

Assume that $\langle X, \hat{\eta}\rangle<0$ and $\langle X, \bar{\eta}\rangle<0$. Notice that $E\left(Y_{\lambda}\right) \backslash 0 \subseteq\left\{X:\left\langle X, N_{\lambda}\right\rangle<0\right\}$, where $N_{\lambda}=\left(v_{\lambda},-1\right)$ and hence, it is enough to show that $\left\langle X, N_{\lambda}\right\rangle \geq 0$.

We first assume that $H\left(v_{\lambda}\right)-H(\bar{v}) \neq 0$ and $H\left(v_{\lambda}\right)-H(\hat{v}) \neq 0$. We will show that we can write

$$
N_{\lambda}=\frac{(1-t)}{H\left(v_{\lambda}\right)-H(\bar{v})} \bar{\eta}+\frac{t}{H\left(v_{\lambda}\right)-H(\hat{v})} \hat{\eta},
$$

with $\frac{(1-t)}{H\left(v_{\lambda}\right)-H(\bar{v})} \leq 0$ and $\frac{t}{H\left(v_{\lambda}\right)-H(\hat{v})} \leq 0$, for some $t$. The above equality holds if and only if

$$
\frac{(1-t)\left(H\left(v_{\lambda}\right) v_{\lambda}-H(\bar{v}) \bar{v}\right)}{H\left(v_{\lambda}\right)-H(\bar{v})}+\frac{t\left(H\left(v_{\lambda}\right) v_{\lambda}-H(\hat{v}) \hat{v}\right)}{H\left(v_{\lambda}\right)-H(\hat{v})}=v_{\lambda},
$$


which holds if and only if

$$
(1-t)\left(v_{\lambda}+\frac{H(\bar{v})\left(v_{\lambda}-\bar{v}\right)}{H\left(v_{\lambda}\right)-H(\bar{v})}\right)+t\left(v_{\lambda}+\frac{H(\hat{v})\left(v_{\lambda}-\hat{v}\right)}{H\left(v_{\lambda}\right)-H(\hat{v})}\right)=v_{\lambda},
$$

which is true if and only if

$$
(\hat{v}-\bar{v})\left\{\frac{(1-t) \lambda H(\bar{v})}{H\left(v_{\lambda}\right)-H(\bar{v})}-\frac{t(1-\lambda) H(\hat{v})}{H\left(v_{\lambda}\right)-H(\hat{v})}\right\}=0 .
$$

Therefore we choose $t$ such that $\frac{(1-t) \lambda H(\bar{v})}{H\left(v_{\lambda}\right)-H(\bar{v})}=\frac{t(1-\lambda) H(\hat{v})}{H\left(v_{\lambda}\right)-H(\hat{v})}$. Since $Q(v)>0$, we have that $\lambda H(\bar{v})>0$ and $(1-\lambda) H(\hat{v})>0$. Then it follows that $\frac{(1-t)}{H\left(v_{\lambda}\right)-H(\bar{v})}$ and $\frac{t}{H\left(v_{\lambda}\right)-H(\hat{v})}$ have both the same sign. From the last identity containing $t$ we obtain

$$
\lambda H(\bar{v})=\frac{t}{H\left(v_{\lambda}\right)-H(\hat{v})}\left\{H\left(v_{\lambda}\right)((1-\lambda) H(\hat{v})+\lambda H(\bar{v}))-H(\bar{v}) H(\hat{v})\right\} .
$$

From 4.10 we get

$$
H\left(v_{\lambda}\right)((1-\lambda) H(\hat{v})+\lambda H(\bar{v}))-H(\bar{v}) H(\hat{v}) \leq 0,
$$

hence $\frac{t}{H\left(v_{\lambda}\right)-H(\hat{v})} \leq 0$, and therefore also $\frac{(1-t)}{H\left(v_{\lambda}\right)-H(\bar{v})} \leq 0$.

Next we assume that $H\left(v_{\lambda}\right)-H(\hat{v})=0$ and $H\left(v_{\lambda}\right)-H(\bar{v}) \neq 0$. From (4.10), this implies that $H\left(v_{\lambda}\right)<H(\bar{v})$. If we write $N_{\lambda}=\frac{1}{H\left(v_{\lambda}\right)-H(\bar{v})} \bar{\eta}+t \hat{\eta}$, then $t=$ $\frac{\lambda H(\bar{v})}{\left(H\left(v_{\lambda}\right)-H(\bar{v})\right)(1-\lambda) H(\hat{v})}$, and so $t<0$.

Finally, if $H\left(v_{\lambda}\right)=H(\hat{v})=H(\bar{v})$, then $\bar{\eta}=\lambda H(\bar{v})(\hat{v}-\bar{v}, 0)$, and $\hat{\eta}=(1-\lambda) H(\bar{v})(\bar{v}-$ $\hat{v}, 0)$. So in this case, both inequalities $\langle X, \hat{\eta}\rangle<0$ and $\langle X, \bar{\eta}\rangle<0$ are impossible.

This proves Claim 3 and hence the proof of the proposition is complete.

Proposition 4.4. Suppose that $u$ is a parallel refractor in $\Omega, x_{0} \in \Omega$, and assume that (4.5) holds from $X_{0}=\left(x_{0}, u\left(x_{0}\right)\right)$. If there exist $Y_{0} \in \sum$ and $\epsilon>0$ such $u(x) \leq \phi\left(x, Y_{0}, X_{0}\right)$ for all $x \in B_{\epsilon}\left(x_{0}\right)$, then $u(x) \leq \phi\left(x, Y_{0}, X_{0}\right)$ for all $x \in \Omega$.

Proof. We define

$$
\partial u\left(x_{0}\right)=\left\{v \in \mathbb{R}^{n}: u(x) \leq u\left(x_{0}\right)+v \cdot\left(x-x_{0}\right)+o\left(\left|x-x_{0}\right|\right) ; \forall x \in B_{\epsilon}\left(x_{0}\right)\right\} .
$$

We prove that $\left\{Y\left(X_{0}, v\right): v \in \partial u\left(x_{0}\right)\right\} \subset F_{u}\left(x_{0}\right)$, where $Y\left(X_{0}, v\right)=X_{0}+s_{X_{0}}(\Lambda(v)) \Lambda(v)$. Since from (2.11) $v=D_{x} \phi(x, Y, X)$ with $Y \in \Sigma$ if and only if $Y=Y(X, v)$, the inclusion is equivalent to show that $\partial u\left(x_{0}\right) \subset\left\{D_{x} \phi\left(x_{0}, Y, X_{0}\right): Y \in F_{u}\left(x_{0}\right)\right\}:=B$. It is then enough to show that the extremal points of $\partial u\left(x_{0}\right)$ are contained in $B$ and that $B$ is convex. Let $v_{0}$ be a extremal point of $\partial u\left(x_{0}\right)$, then there exist $x_{n} \rightarrow x_{0}$ with $u$ differentiable at $x_{n}$ and $v_{n}=D u\left(x_{n}\right) \rightarrow v_{0}$, see [?, Theorem 2.5.1]. Let 
$X_{n}=\left(x_{n}, u\left(x_{n}\right)\right)$ and let $Y_{n} \in F_{u}\left(x_{n}\right)$. Since $u$ is differentiable at $x_{n}$ it follows that $Y_{n}=Y\left(X_{n}, v_{n}\right)$ and then $Y_{n} \rightarrow Y\left(X_{0}, v_{0}\right):=Y_{0}$. We have that $u(x) \leq \phi\left(x, Y_{n}, X_{n}\right)$ for all $x \in \Omega$. Letting $n \rightarrow \infty$ yields $u(x) \leq \phi\left(x, Y_{0}, X_{0}\right)$ for all $x \in \Omega$, i.e., $Y_{0} \in F_{u}\left(x_{0}\right)$ and $D_{x} \phi\left(x_{0}, Y_{0}, X_{0}\right)=v_{0}$.

To show that $B$ is convex, let $Y_{1}, Y_{2} \in F_{u}\left(x_{0}\right)$ and let $v_{i}=D_{x} \phi\left(x_{0}, Y_{i}, X_{0}\right), i=1,2$. Consider $v_{\lambda}=(1-\lambda) v_{1}+\lambda v_{2}$ and $Y_{\lambda}=Y\left(X_{0}, v_{\lambda}\right)$. Since $v_{\lambda}=D_{x} \phi\left(x_{0}, Y_{\lambda}, X_{0}\right)$, we need to show that $Y_{\lambda} \in F_{u}\left(x_{0}\right)$. We have

$$
u(x) \leq \min \left\{\phi\left(x, Y_{1}, X_{0}\right), \phi\left(x, Y_{2}, X_{0}\right)\right\} \leq \phi\left(x, Y_{\lambda}, X_{0}\right)
$$

for all $x \in \Omega$ by Proposition 4.3 . This completes the proof.

4.3. Main result. We will now prove our main theorem.

Theorem 4.5. Suppose $u$ is a parallel refractor, and the target $\Sigma$ is regular from $X^{\star}=$ $\left(x^{\star}, u\left(x^{\star}\right)\right)$ in the sense of Definition 3.1. Let $C, C_{1}, C_{2}$ and $\delta$ be the constants of Lemma 4.2 and $M$ a constant depending on $C$ and $C_{1}$ to be chosen. Then, if $\hat{x}, \bar{x} \in B_{\frac{\delta}{2}}\left(x^{\star}\right)$ and $\bar{Y} \in F_{u}(\bar{x}), \hat{Y} \in F_{u}(\hat{x})$ are such that

$$
\frac{|\bar{Y}-\hat{Y}|}{|\bar{x}-\hat{x}|} \geq \max \left\{1,\left(\frac{2 M}{\delta}\right)^{2},\left(\frac{2 M}{C_{2}}\right)^{2}\right\}
$$

then there exists $x_{0}$ on the straight segment $\overline{\bar{x} \hat{x}}$ such that, setting $X_{0}^{\star}=\left(x_{0}, u\left(x_{0}\right)\right)$, we have

$$
N_{\mu}\left(\left\{Y(\lambda) \in[\bar{Y}, \hat{Y}]_{X_{0}^{\star}}: \lambda \in[1 / 4,3 / 4]\right\}\right) \cap \Sigma \subseteq F_{u}\left(B_{\eta}\left(x_{0}\right)\right)
$$

with $\mu=|\bar{Y}-\hat{Y}|^{\frac{3}{2}}|\bar{x}-\hat{x}|^{\frac{1}{2}}$ and $\eta=M \frac{|\bar{x}-\hat{x}|^{\frac{1}{2}}}{|\bar{Y}-\hat{Y}|^{\frac{1}{2}}}$.

Proof. From the assumption, we have $|\bar{Y}-\hat{Y}| \geq|\bar{x}-\hat{x}|$ and so Lemma 4.2 is applicable. Let the point $x_{0}$ and the constant $C_{2}$ be as in that lemma.

Fix $Y \in N_{\mu}\left(\left\{Y(\lambda) \in[\bar{Y}, \hat{Y}]_{X_{0}^{\star}}: \lambda \in\left[\frac{1}{4}, \frac{3}{4}\right]\right\}\right) \cap \Sigma$. So, there exists $Y(\lambda) \in[\bar{Y}, \hat{Y}]_{X_{0}^{\star}}$ with $\lambda \in\left[\frac{1}{4}, \frac{3}{4}\right]$ such that $|Y(\lambda)-Y|<\mu$.

We then have from Lemma 4.2 that

$$
\begin{aligned}
u(x)-\phi\left(x, Y, X_{0}^{\star}\right) & \leq C|\bar{Y}-\hat{Y} \| \bar{x}-\hat{x}|+C|Y(\lambda)-Y|\left|x-x_{0}\right|-C_{1} \lambda(1-\lambda)|\bar{Y}-\hat{Y}|^{2}\left|x-x_{0}\right|^{2} \\
& \leq C|\bar{Y}-\hat{Y} \| \bar{x}-\hat{x}|+C \mu\left|x-x_{0}\right|-\frac{C_{1}}{16}|\bar{Y}-\hat{Y}|^{2}\left|x-x_{0}\right|^{2},
\end{aligned}
$$

for all $x \in \Omega$ such that $\left|x-x_{0}\right|<C_{2}$.

The right hand side in the last inequality is strictly negative for all $x \in \Omega$, with $\left|x-x_{0}\right| \geq \eta$, if we choose $\eta>\frac{8 C \mu+4 \sqrt{4 C^{2} \mu^{2}+C C_{1}|\bar{Y}-\hat{Y}|^{3}|\bar{x}-\hat{x}|}}{C_{1}|\bar{Y}-\hat{Y}|^{2}}$. We pick $\mu=|\bar{Y}-\hat{Y}|^{\frac{3}{2}}|\bar{x}-\hat{x}|^{\frac{1}{2}}$, and $\eta:=M \frac{|\bar{x}-\hat{x}|^{\frac{1}{2}}}{|\bar{Y}-\hat{Y}|^{\frac{1}{2}}}$ with $M:=M\left(C, C_{1}\right)=M\left(X^{\star}\right)$. In 
fact we let $M=2\left(\frac{8 C+\sqrt{4 C^{2}+C C_{1}}}{C_{1}}\right)$ Since $x_{0} \in B_{\frac{\delta}{2}}\left(x^{\star}\right)$, it follows from (4.11) that $B_{\eta}\left(x_{0}\right) \subset B_{\delta}\left(x^{\star}\right) \subset \Omega$ and $\eta \leq \frac{C_{2}}{2}$. Therefore $u(x)-\phi\left(x, Y, X_{0}^{\star}\right)<0$ for all $x \in\left(\Omega \cap B_{C_{2}}\left(x_{0}\right)\right) \backslash B_{\eta}\left(x_{0}\right)$.

We now show $Y \in F_{u}\left(B_{\eta}\left(x_{0}\right)\right)$. Notice that by definition of $X_{0}^{\star}$ we have $u\left(x_{0}\right)=$ $\phi\left(x_{0}, Y, X_{0}^{\star}\right)$.

Let $G_{u}$ denote the graph of $u$ in $B_{C_{2}}\left(x_{0}\right)$, that is, $G_{u}=\left\{(x, u(x)): x \in \Omega \cap B_{C_{2}}\left(x_{0}\right)\right\}$. Consider

$$
\inf \left\{c(X, Y)-c\left(X_{0}^{\star}, Y\right): X \in G_{u}\right\},
$$

recalling that $c(X, Y)=|X-Y|+k\left(x_{n+1}-y_{n+1}\right)$. We claim that $c(X, Y)-c\left(X_{0}^{\star}, Y\right) \geq 0$ for $X=(x, u(x))$ with $\left|x-x_{0}\right| \geq \eta$. Indeed, let $\bar{X}=\left(x, \phi\left(x, Y, X_{0}^{\star}\right)\right)$ and notice that $c\left(X_{0}^{\star}, Y\right)=c(\bar{X}, Y)$. Since $\Sigma \subset \mathcal{T}$, with $\mathcal{T}$ given by (2.6), we have $X \in E^{-}(Y, c(X, Y))$. Since $u(x)-\phi\left(x, Y, X_{0}^{\star}\right) \leq 0$ for $\left|x-x_{0}\right| \geq \eta$, we have that $X$ is below $\bar{X}$, and therefore we must have $c(X, Y) \geq c(\bar{X}, Y)$, and the claim follows. Therefore, the infimum in 4.12) is attained at some point $\tilde{X}=(\tilde{x}, u(\tilde{x}))$ with $\tilde{x} \in B_{\eta}\left(x_{0}\right)$.

We show that $Y \in F_{u}(\tilde{x})$. Indeed, we have $c(X, Y) \geq c(\tilde{X}, Y)$ for all $X \in G_{u}$. Writing $X=(x, u(x))$ we have that $\left(|x-y|^{2}+\left(y_{n+1}-u(x)\right)^{2}\right)^{1 / 2}-k\left(y_{n+1}-u(x)\right) \geq c(\tilde{X}, Y)$ and noticing that $y_{n+1} \geq u(x)$, we get $u(x) \leq y_{n+1}-\frac{k c(\tilde{X}, Y)}{1-k^{2}}-\sqrt{\frac{c(\tilde{X}, Y)^{2}}{\left(1-k^{2}\right)^{2}}-\frac{|x-y|^{2}}{1-k^{2}}}=$ $\phi(x, Y, \tilde{X})$ for $x \in \Omega \cap B_{C_{2}}\left(x_{0}\right)$. Since $\eta<C_{2} / 2$, we have that $B_{C_{2} / 2}(\tilde{x}) \subset B_{C_{2}}\left(x_{0}\right)$. We therefore obtain the local estimate $u(x) \leq \phi(x, Y, \tilde{X})$ for all $x \in B_{\epsilon}(\tilde{x})$, with $\epsilon$ small.

Since $\tilde{x} \in B_{\delta}\left(x^{\star}\right)$, it follows that $\tilde{X}=(\tilde{x}, u(\tilde{x})) \in U_{X^{\star}}$. In particular (4.5) holds at $\tilde{X}$ and hence, from Proposition 4.4 we then obtain that this estimate holds in $\Omega$ and the proof of the theorem is complete.

4.4. A property of refractors. Let $\sigma$ denote the Borel measure given on the target $\Sigma$ and let $\mu$ be a Borel measure in $\Omega$. We say $x \in \mathcal{T}_{u}(Y)$ if and only if $Y \in F_{u}(x)$, where $F_{u}$ is defined by (4.1). Assuming $\mu=f d x$ with $f \in L^{1}(\Omega)$ and the energy conservation condition

$$
\mu(\Omega)=\sigma(\Sigma),
$$

it is proved in [?] the existence of a refractor $u$ such that

$$
\mu\left(\mathcal{T}_{u}\left(E^{\star}\right)\right)=\sigma\left(E^{\star}\right), \quad \text { for all Borel subsets } E^{\star} \subset \Sigma .
$$

The purpose of this subsection is to show the following proposition.

Proposition 4.6. Suppose $u$ is a refractor solving (4.13), and define

$$
S=\left\{x \in \Omega \text { : there exists } \bar{x} \neq x, \bar{x} \in \Omega \text {, such that } F_{u}(x) \cap F_{u}(\bar{x}) \neq \emptyset\right\} .
$$

Let us assume the following conditions on $\Sigma$ and $\sigma$ :

(a) $\Sigma$ is the graph of a $C^{1}$ function, say $\Sigma=\left\{(y, \psi(y)): y \in \Omega^{\star}\right\}$ with $\Omega^{*}$ some domain in $\mathbb{R}^{n}$; 
(b) Given $Y \in \Sigma$, let $T_{Y}$ denote the tangent plane to $\Sigma$ at $Y$. Assume that for each $Y \in \Sigma$ and for each $X \in C_{\Omega}$, the line $\{X+s(Y-X), s \in R\}$ is not contained in $T_{Y}$, that is, this line intersects $T_{Y}$ only at the point $Y$. .

(c) if $E \subset \sum$ with $\left|\left\{y \in \Omega^{*}:(y, \psi(y)) \in E\right\}\right|=0$, then $\sigma(E)=0$.

Then $\mu(S)=0$ and we have the inequality

$$
\sigma\left(F_{u}(B)\right) \leq \mu(B), \quad \text { for all balls } B \subset \Omega .
$$

Proof. We first notice that $F_{u}(B)$ is a Borel set for each closed ball $B$. Because if $K \subseteq \Omega$ is closed, then $F_{u}(K)$ is closed in $\Sigma$. In fact, let $Y_{k} \in F_{u}\left(x_{k}\right)$ with $x_{k} \in K$ and assume $Y_{k} \rightarrow Y$ with $Y \in \Sigma$. There exists a subsequence $x_{k_{j}} \rightarrow \bar{x}$ for some $\bar{x} \in K$. Setting $X_{k_{j}}=\left(x_{k_{j}}, u\left(x_{k_{j}}\right)\right)$, we have $X_{k_{j}} \rightarrow \bar{X}$ where $X=(\bar{x}, u(\bar{x}))$. Since $u(x) \leq \phi\left(x, Y_{k}, X_{k}\right)$ for all $x \in \Omega$ and for all $k$, we have $u(x) \leq \phi(x, Y, \bar{X})$. Therefore $Y \in F_{u}(K)$.

Let us assume for a moment that $\mu(S)=0$. It is easy to see that that $\mathcal{T}_{u}\left(F_{u}(B)\right) \subseteq$ $B \cup S$, and therefore, (4.14) follows.

To prove that $\mu(S)=0$, let us consider the set

$$
S^{\star}=\left\{Y \in \Sigma: \exists x, \bar{x} \in \Omega, x \neq \bar{x} \quad Y \in F_{u}(x) \cap F_{u}(\bar{x})\right\} .
$$

We have that $\mathcal{T}_{u}\left(S^{\star}\right)=S$ and therefore $\mu(S)=\sigma\left(S^{\star}\right)$. Under the assumptions (a), (b) and (c) above, we are going to show that $\sigma\left(S^{\star}\right)=0$. To this end, we define $u^{\star}: \Omega^{\star} \rightarrow R$ by

$$
u^{\star}(y)=\inf \left\{c(X, Y): X \in G_{u}, Y=(y, \psi(y))\right\}
$$

where $G_{u}$ is the graph of $u$. We claim $u^{\star}$ is Lipschitz in $\Omega^{\star}$. Indeed, say $u^{\star}\left(y_{0}\right)=$ $c\left(X_{0}, Y_{0}\right)$ and $u^{\star}\left(y_{1}\right)=c\left(X_{1}, Y_{1}\right)$, then $u^{\star}\left(y_{1}\right)-u^{\star}\left(y_{0}\right) \leq c\left(X_{0}, Y_{1}\right)-c\left(X_{0}, Y_{0}\right) \leq$ $C\left|Y_{1}-Y_{0}\right| \leq C\left|y_{1}-y_{0}\right|$, since $\psi$ is Lipschitz. Similarly, we get the other inequality.

Now fix $Y_{0} \in S^{\star}$, so $Y_{0} \in F_{u}(x) \cap F_{u}(\bar{x})$ with $x \neq \bar{x}$. Write $Y_{0}=\left(y_{0}, \psi\left(y_{0}\right)\right)$ with $y_{0} \in \Omega^{\star}, X=(x, u(x))$ and $\bar{X}=(\bar{x}, u(\bar{x}))$. We claim that $u^{\star}$ is not differentiable at $y_{0}$. Suppose by contradiction that $u^{\star}$ is differentiable at $y_{0}$. It is easy to see that $u^{\star}\left(y_{0}\right)=c\left(X, Y_{0}\right)$ and also $u^{\star}\left(y_{0}\right)=c\left(\bar{X}, Y_{0}\right)$. From $u^{\star}\left(y_{0}\right)=c\left(X, Y_{0}\right)$, it follows that $u^{\star}(y) \leq c(X, Y)$ for all $y \in \Omega^{\star}$ with equality at $y_{0}$. Hence we have

$$
D u^{\star}\left(y_{0}\right)=D_{y}(c(X,(y, \psi(y))))\left(y_{0}\right)=\frac{y_{0}-x+\left(\psi\left(y_{0}\right)-x_{n+1}\right) D \psi\left(y_{0}\right)}{\left|Y_{0}-X\right|}-k D \psi\left(y_{0}\right) .
$$

Also from $u^{\star}\left(y_{0}\right)=c\left(\bar{X}, Y_{0}\right)$, we deduce that

$$
D u\left(y_{0}\right)=D_{y}(c(\bar{X},(y, \psi(y))))\left(y_{0}\right)=\frac{y_{0}-\bar{x}+\left(\psi\left(y_{0}\right)-\bar{x}_{n+1}\right) D \psi\left(y_{0}\right)}{\left|Y_{0}-\bar{X}\right|}-k D \psi\left(y_{0}\right) .
$$

${ }^{+}$This condition is implied by the visibility condition in Lemma 2.1 because $Y \in \Sigma$ and some $X_{0} \in C_{\Omega}$ the line joining $Y$ and $X_{0}$ is contained in $T_{Y}$, then there is ball $B$ centered at $X_{0}$ with $B \subset C_{\Omega}$. By the visibility condition the convex hull $C$ of $Y$ and $B$ intersects $\Sigma$ only at $Y$. But then the line joining $Y$ and $X_{0}$ is contained in $C$ and $T_{Y}$. Therefore $\Sigma$ is not differentiable at $Y$. 
This implies that $\frac{y_{0}-x+\left(\psi\left(y_{0}\right)-x_{n+1}\right) D \psi\left(y_{0}\right)}{\left|Y_{0}-X\right|}=\frac{y_{0}-\bar{x}+\left(\psi\left(y_{0}\right)-\bar{x}_{n+1}\right) D \psi\left(y_{0}\right)}{\left|Y_{0}-\bar{X}\right|}$. Let us set $\Gamma=\frac{X-Y_{0}}{\left|X-Y_{0}\right|}=\left(\xi, \xi_{n+1}\right)$ and $\bar{\Gamma}=\frac{\bar{X}-Y_{0}}{\left|\bar{X}-Y_{0}\right|}=\left(\bar{\xi}, \bar{\xi}_{n+1}\right)$. So $\bar{\xi}+\bar{\xi}_{n+1} D \psi\left(y_{0}\right)=$ $\xi+\xi_{n+1} D \psi\left(y_{0}\right)$ and hence $\bar{\xi}-\xi=D \psi\left(y_{0}\right)\left(\xi_{n+1}-\bar{\xi}_{n+1}\right)$.

If $\xi_{n+1}=\bar{\xi}_{n+1}$, then $\xi=\bar{\xi}$. Hence $\Gamma=\bar{\Gamma}$ but this implies that $X=\bar{X}$, since $X, \bar{X} \in E^{-}\left(Y_{0}, b\right)$ where $b=c\left(X, Y_{0}\right)=c\left(\bar{X}, Y_{0}\right)$. Since by assumption $X \neq \bar{X}$, we obtain a contradiction.

Therefore, $\xi_{n+1} \neq \bar{\xi}_{n+1}$ and so we can write $D \psi\left(y_{0}\right)=\frac{\bar{\xi}-\xi}{\xi_{n+1}-\bar{\xi}_{n+1}}$. We claim that the line $L:=\left\{Y_{0}+s\left(\frac{\Gamma}{2}+\frac{\bar{\Gamma}}{2}\right): s \in R\right\}$ is contained in $T_{Y_{0}}$. Indeed, if $Y=Y_{0}+s\left(\frac{\Gamma}{2}+\frac{\bar{\Gamma}}{2}\right)$, then a simple calculation shows that $\left\langle Y-Y_{0},\left(-D \psi\left(y_{0}\right), 1\right)\right\rangle=0$. On the other hand, the line $L$ clearly intersects $C_{\Omega}$. We then obtain a contradiction with the assumption (b) above, and therefore $u^{\star}$ is not differentiable at $y_{0}$.

If we set $P^{\star}=\left\{y \in \Omega^{\star}:(y, \psi(y)) \in S^{\star}\right\}$, then we proved that $y \in P^{\star}$ implies that $u^{\star}$ is not differentiable at $y$. Since $u^{\star}$ is Lipschitz in $\Omega^{\star}$, we get $\left|P^{\star}\right|=0$. Therefore from (c) we obtain $\sigma\left(S^{\star}\right)=0$ which completes the proof of the proposition.

\section{HöldER CONTINUITY OF THE GRADIENT OF THE REFRACTOR}

We introduce the following local condition at $X_{0} \in C_{\Omega}$ between the measure $\sigma$ and target $\Sigma$ : There exist a neighborhood $U_{X_{0}}$ and a constant $\hat{C}>0$ depending on $X_{0}$ such that

$$
\sigma\left(N_{\mu}\left(\left\{[\bar{Y}, \hat{Y}]_{Z}: \lambda \in[1 / 4,3 / 4]\right\}\right) \cap \Sigma\right) \geq \hat{C} \mu^{n-1}|\bar{Y}-\hat{Y}|
$$

for any $\bar{Y}, \hat{Y} \in \Sigma, Z \in U_{X_{0}}$ and for all $\mu>0$ small (depending on $X_{0}$ ). Here $N_{\mu}(E)$ denotes the $\mu$ - neighborhood of the set $E$ in $R^{n+1}$.

Theorem 5.1. Suppose $u$ is a parallel refractor, the target $\Sigma$ is regular from $X^{\star}=$ $\left(x^{\star}, u\left(x^{\star}\right)\right)$ in the sense of Definition 3.1. and there exist constants $C_{0}>0$ and $1 \leq q<$ $\frac{n}{n-1}$ such that

$$
\sigma\left(F_{u}\left(B_{\eta}\right)\right) \leq C_{0} \eta^{n / q}
$$

for all balls $B_{\eta} \subseteq \Omega$. Suppose in addition that the local condition (5.1) is satisfied at $X^{\star}$.

Then there exist constants $\delta, M>0$ and $C_{2}>0$ depending on $X^{\star}$, such that if $\hat{x}, \bar{x} \in B_{\frac{\delta}{2}}\left(x^{\star}\right), \bar{Y} \in F_{u}(\bar{x}), \hat{Y} \in F_{u}(\hat{x})$ are such that

$$
\frac{|\bar{Y}-\hat{Y}|}{|\bar{x}-\hat{x}|} \geq \max \left\{1,\left(\frac{2 M}{\delta}\right)^{2},\left(\frac{2 M}{C_{2}}\right)^{2}\right\},
$$


then we have $|\bar{Y}-\hat{Y}| \leq C_{1}|\bar{x}-\hat{x}|^{\alpha}$ with $\alpha=\frac{\frac{n}{2 q}-\frac{n-1}{2}}{1+\frac{3}{2}(n-1)+\frac{n}{2 q}}$, where $C_{1}$ depends only on $C_{0}$ and $\hat{C}$ in (5.1), and therefore from $X^{\star}$.

Proof. From Theorem 4.5 we have $N_{\mu}\left(\left\{Y(\lambda) \in[\bar{Y}, \hat{Y}]_{X_{0}^{\star}}: \lambda \in\left[\frac{1}{4}, \frac{3}{4}\right]\right\}\right) \cap \Sigma \subseteq F_{u}\left(B_{\eta}\left(x_{0}\right)\right)$ where $\mu=|\bar{Y}-\hat{Y}|^{\frac{3}{2}}|\bar{x}-\hat{x}|^{\frac{1}{2}}$ and $\eta=M \frac{|\bar{x}-\hat{x}|^{\frac{1}{2}}}{|\bar{Y}-\hat{Y}|^{\frac{1}{2}}}$. Therefore from (5.1) and (5.2), we obtain $|\bar{Y}-\hat{Y}|^{1+\frac{3}{2}(n-1)+\frac{n}{2 q}} \leq C|\bar{x}-\hat{x}|^{\frac{n}{2 q}-\frac{n-1}{2}}$ and the theorem follows.

Under all previous hypotheses on the target, we now show interior $C^{1, \alpha}$ estimates.

Theorem 5.2. With the assumptions of Theorem 5.1, there exist positive constants $\delta$ and $C$, depending on $X^{\star}$, such that $u \in C^{1, \alpha}\left(B_{\delta}\left(x^{\star}\right)\right)$ with $|D u(\bar{x})-D u(\hat{x})| \leq C|\bar{x}-\hat{x}|^{\alpha}$ for all $\hat{x}, \bar{x} \in B_{\delta}\left(x^{\star}\right)$.

Proof. By Theorem 5.1 we get that $F_{u}(x)$ is a singleton for each $x \in B_{\delta}\left(x^{\star}\right)$. Take $\bar{x} \in B_{\delta / 2}\left(x^{\star}\right)$.

We first show that if $\bar{Y} \in F_{u}(\bar{x})$, then $u$ is differentiable at $\bar{x}$, and $D_{i} u(\bar{x})=$ $D_{i} \phi(\bar{x}, \bar{Y}, \bar{X})$ where $\bar{X}=(\bar{x}, u(\bar{x}))$.

For $0<h<\delta / 2$ we have

$$
\begin{aligned}
\frac{u\left(\bar{x}+h e_{i}\right)-u(\bar{x})}{h}-D_{i} \phi(\bar{x}, \bar{Y}, \bar{X}) & \leq \frac{\phi\left(\bar{x}+h e_{i}, \bar{Y}, \bar{X}\right)-\phi(\bar{x}, \bar{Y}, \bar{X})}{h}-D_{i} \phi(\bar{x}, \bar{Y}, \bar{X}) \\
& =D_{i} \phi\left(\bar{x}+\tilde{h} e_{i}, \bar{Y}, \bar{X}\right)-D_{i} \phi(\bar{x}, \bar{Y}, \bar{X}), \quad \text { for some } 0 \leq \tilde{h} \leq h \\
& =D_{i, i} \phi\left(\bar{x}+\hat{h} e_{i}, \bar{Y}, \bar{X}\right) \tilde{h},
\end{aligned}
$$

for some $0 \leq \hat{h} \leq \tilde{h}$. Hence $\frac{u\left(\bar{x}+h e_{i}\right)-u(\bar{x})}{h}-D_{i} \phi(\bar{x}, \bar{Y}, \bar{X}) \leq C h$.

To prove the inequality in the opposite direction, let $\hat{x}=\bar{x}+h e_{i}, \hat{Y} \in F_{u}(\hat{x})$, and $\hat{X}=(\hat{x}, u(\hat{x}))$. We have that

$$
\begin{aligned}
D_{i} \phi(\bar{x}, \bar{Y}, \bar{X})-\frac{(u(\hat{x})-u(\bar{x}))}{h} & \leq D_{i} \phi(\bar{x}, \bar{Y}, \bar{X})-\frac{(\phi(\hat{x}, \hat{Y}, \hat{X})-\phi(\bar{x}, \hat{Y}, \hat{X}))}{h} \\
& =D_{i} \phi(\bar{x}, \bar{Y}, \bar{X})-D_{i} \phi(\tilde{x}, \hat{Y}, \hat{X})
\end{aligned}
$$

for some $\tilde{x} \in[\bar{x}, \hat{x}]$. From the estimates for the derivatives of $\phi$ from Subsection (2.4), we can also write

$$
\begin{aligned}
& D_{i} \phi(\bar{x}, \bar{Y}, \bar{X})-D_{i} \phi(\tilde{x}, \hat{Y}, \hat{X}) \\
& =D_{i} \phi(\bar{x}, \bar{Y}, \bar{X})-D_{i} \phi(\tilde{x}, \bar{Y}, \bar{X})+D_{i} \phi(\tilde{x}, \bar{Y}, \bar{X})-D_{i} \phi(\tilde{x}, \hat{Y}, \bar{X})+D_{i} \phi(\tilde{x}, \hat{Y}, \bar{X})-D_{i} \phi(\tilde{x}, \hat{Y}, \hat{X}) \\
& \leq C(|\bar{x}-\hat{x}|+|\bar{Y}-\hat{Y}|+|\bar{X}-\hat{X}|),
\end{aligned}
$$


with $C$ a structural constant. On the other hand, since $u$ is Lipschitz (with a constant depending only on structure), we have $|\bar{X}-\hat{X}| \leq|\bar{x}-\hat{x}|+|u(\bar{x})-u(\hat{x})| \leq$ $C|\bar{x}-\hat{x}|$. In addition, using Theorem 5.1, we get that $|\bar{Y}-\hat{Y}| \leq C \max \{\mid \bar{x}-$ $\left.\hat{x}|,| \bar{x}-\left.\hat{x}\right|^{\alpha}\right\} \leq C h^{\alpha}$ where $C$ is a structural constant depending also on $X^{\star}$. This

yields $\frac{u\left(\bar{x}+h e_{i}\right)-u(\bar{x})}{h}-D_{i} \phi(\bar{x}, \bar{Y}, \bar{X}) \geq-C h^{\alpha}$, completing the proof that $D_{i} u(\bar{x})=$ $D_{i} \phi(\bar{x}, \bar{Y}, \bar{X})$.

We finally prove that $u \in C^{1, \alpha}\left(B_{\delta / 2}\left(x^{\star}\right)\right)$. Let $\bar{x}, \hat{x} \in B_{\frac{\delta}{2}}\left(x^{\star}\right), \bar{Y} \in F_{u}(\bar{x}), \hat{Y} \in F_{u}(\hat{x})$, and set $\bar{X}=(\bar{x}, u(\bar{x})), \hat{X}=(\hat{x}, u(\hat{x}))$. We then have $\left|D_{i} u(\bar{x})-D_{i} u(\hat{x})\right|=\mid D_{i} \phi(\bar{x}, \bar{Y}, \bar{X})-$ $D_{i} \phi(\hat{x}, \hat{Y}, \hat{X})|\leq| D_{i} \phi(\bar{x}, \bar{Y}, \bar{X})-D_{i} \phi(\hat{x}, \bar{Y}, \bar{X})|+| D_{i} \phi(\hat{x}, \bar{Y}, \bar{X})-D_{i} \phi(\hat{x}, \hat{Y}, \bar{X})|+| D_{i} \phi(\hat{x}, \hat{Y}, \bar{X})-$ $D_{i} \phi(\hat{x}, \hat{Y}, \hat{X})|\leq C\{|\bar{x}-\hat{x}|+|\bar{Y}-\hat{Y}|+|\bar{X}-\hat{X}|\} \leq C| \bar{x}-\left.\hat{x}\right|^{\alpha}$, with a structural constant $C$ depending also on $X^{\star}$.

Corollary 5.3. Suppose $u$ is a refractor solving (4.13), conditions (a), (b), and (c) from Proposition 4.6 hold, and $\mu=f d x$ with $f \in L^{p}(\Omega)$ for some $p>n$. Suppose that the target $\Sigma$ is regular from $X^{\star}=\left(x^{\star}, u\left(x^{\star}\right)\right)$ in the sense of Definition 3.1. and the local condition (5.1) is satisfied at $X^{\star}$. Then there exist $\delta$ and $C$ depending on $X^{\star}$ such that $u \in C^{1, \alpha}\left(B_{\delta}\left(x^{\star}\right)\right)$ with $\alpha$ given in Theorem 5.1, and $1 / p+1 / q=1$.

Proof. By Hölder's inequality $\mu(B) \leq\|f\|_{p}|B|^{1 / q}$, for all balls $B \subset \Omega$. Therefore from (4.14) we obtain condition (5.2), and the corollary follows.

\section{EXAMPLE OF A TARGET FOR REFRACTION}

If we assume the target set $\Sigma$ is given by the graph of a $C^{2}$ function $y_{n+1}=\psi(y)$, then we are going to find a condition on $\psi$ so that (3.6) holds locally. We will see that for (3.6) to hold, the graph of the target needs to satisfy a quantitative condition, see (6.3). As in Subsection 4.2, we set $H(v, X)=s_{X}(\Lambda(v)) Q(v)$. We have for $Y \in \Sigma$ that $Y=X+s_{X}(\Lambda(v))(-Q(v) v, Q(v)+\kappa)$. Then we can write $y_{n+1}=x_{n+1}+s_{X}(\Lambda(v))(Q(v)+k)=\psi\left(x-s_{X}(\Lambda(v)) Q(v) v\right)$. We therefore get that $H$ satisfies the implicit equation

$$
x_{n+1}+H(v, X)\left(\frac{Q(v)+\kappa}{Q(v)}\right)=\psi(x-H(v, X) v) .
$$

Proceeding as in Subsection 4.2 , setting $G=1 / H$ we need to check that

$$
\left\langle D^{2} G(0, X) \xi, \xi\right\rangle<0
$$

for all $|\xi|=1$. We will prove (6.2) for $X=0$ and by continuity (6.2) will hold for $X$ in a neighborhood of $X=0$. Indeed, we next compute $D_{i j} G(0,0)$ in terms of $\psi$. Since $G=1 / H$, we first proceed to calculate the derivatives of $H$. From $(2.1), Q(v)=$ $\frac{\sqrt{1+\left(1-\kappa^{2}\right)|v|^{2}}-\kappa}{1+|v|^{2}}$ and so $Q(0)=1-\kappa, D_{i} Q(0)=0$ and $D_{i j} Q(0)=-\delta_{i j}(1-\kappa)^{2}$. 
From (6.1), we get $H(0,0)=(1-\kappa) \psi(0), D_{i} H(0,0)=-(1-\kappa)^{2} D_{i} \psi(0) \psi(0)$, and

$$
D_{i j} H(0,0)=(1-\kappa)^{3} \psi(0)\left(\frac{-\kappa}{1-\kappa} \delta_{i j}+2 D_{i} \psi(0) D_{j} \psi(0)+D_{i j} \psi(0) \psi(0)\right) .
$$

Now, noticing that $D_{i j} G=H^{-3}\left(2 D_{i} H D_{j} H-H D_{i j} H\right)$ and inserting the above expressions yields

$$
D_{i j} G(0,0)=\frac{1-\kappa}{\psi(0)}\left(\frac{\kappa}{1-\kappa} \delta_{i j}-\psi(0) D_{i j} \psi(0)\right) .
$$

We therefore obtain that

$$
\left\langle D^{2} G(0,0) \xi, \xi\right\rangle=\frac{1-\kappa}{\psi(0)}\left(\frac{\kappa}{1-\kappa}-\psi(0)\left\langle D^{2} \psi(0) \xi, \xi\right\rangle\right) .
$$

Thus 6.2) is equivalent to

$$
\frac{\kappa}{1-\kappa}<\psi(0)\left\langle D^{2} \psi(0) \xi, \xi\right\rangle,
$$

for all unit vectors $\xi$.

We have then proved that for $\Sigma$ the graph of $\psi$, and for $X_{0}=(0,0), Y_{0}=(0, \psi(0))$, the condition $\left.\frac{d^{2}}{d \epsilon^{2}}\left\langle D^{2} \phi\left(x_{0}, Y_{\epsilon}, X_{0}\right) \eta, \eta\right\rangle\right|_{\epsilon=0}<0$ holds for all unit vectors $\xi \perp \eta$, provided (6.3) holds. By continuity we have that $\left.\frac{d^{2}}{d \epsilon^{2}}\left\langle D^{2} \phi(x, Y(\epsilon), X) \eta, \eta\right\rangle\right|_{\epsilon=0} \leq$ $-C|\xi|^{2}|\eta|^{2}$ for all vectors $\xi \perp \eta$ and for all $X$ close to $X_{0}$ and $Y$ close to $Y_{0}$.

Remark 6.1. We show that if the target $\Sigma$ is a horizontal plane, then (3.1) does not hold when $n \geq 2$. Indeed, we show it is not true that $\phi\left(x, Y(\lambda), X_{0}\right) \geq$ $\min \left\{\phi\left(x, \bar{Y}, X_{0}\right), \phi\left(x, \hat{Y}, X_{0}\right)\right\}$. In $\mathbb{R}^{3}$, let $\bar{Y}=(0,-1,0), \hat{Y}=(0,1,0), \bar{\phi}(x, y)=-\frac{k b}{1-k^{2}}-$ $\left(\frac{b^{2}}{\left(1-k^{2}\right)^{2}}-\frac{x^{2}+(y+1)^{2}}{1-k^{2}}\right)^{1 / 2}$ and $\hat{\phi}(x, y)=-\frac{k b}{1-k^{2}}-\left(\frac{b^{2}}{\left(1-k^{2}\right)^{2}}-\frac{x^{2}+(y-1)^{2}}{1-k^{2}}\right)^{1 / 2}$, with $b$ sufficiently large. Let $X_{0}=(0,0, \bar{\phi}(0,0))=(0,0, \hat{\phi}(0,0))$ and we have $\bar{\phi}(x, y)=\phi\left((x, y), \bar{Y}, X_{0}\right) \hat{\phi}(x, y)=\phi\left((x, y), \hat{Y}, X_{0}\right)$. We also have that $\Lambda(v(\lambda))$ is on the two dimensional plane $x=0$, and so $[\bar{Y}, \hat{Y}]_{X_{0}}=[\bar{Y}, \hat{Y}]$ is the straight segment from $\bar{Y}$ to $\hat{Y}$. We have $Y(1 / 2)=(0,0,0)$. Let $\phi_{0}(x, y):=\phi\left((x, y), Y(1 / 2), X_{0}\right)=$ $-\frac{k b_{0}}{1-k^{2}}-\left(\frac{b_{0}^{2}}{\left(1-k^{2}\right)^{2}}-\frac{\left(x^{2}+y^{2}\right)}{1-k^{2}}\right)^{1 / 2}$, where $b_{0}$ is chosen so that $\left.\phi_{0}(0,0)=\bar{\phi}(0,0)\right)=$ $\hat{\phi}(0,0)$. That is, $b_{0}=\frac{k b+\left(b^{2}-\left(1-k^{2}\right)\right)^{\frac{1}{2}}}{1+k}$. We claim that $\phi_{0}(x, 0)<\bar{\phi}(x, 0)=\hat{\phi}(x, 0)$ for $x \neq 0, x$ small. Let $g(x)=\phi_{0}(x, 0)$ and $h(x)=\bar{\phi}(x, 0)$. Then one can check that $(g-h)^{\prime}(0)=0$ and $(g-h)^{\prime \prime}(0)<0$ which gives the claim. 


\section{ON THE DEFINITION OF REFRACTOR}

We can define refractor with ellipsoids touching $u$ from below, that is, the ellipsoids enclose $u$. In fact, we can define analogously to (4.1)

$$
\tilde{F}_{u}\left(x_{0}\right)=\left\{Y \in \Sigma: u(x) \geq \phi\left(x, Y, X_{0}\right) \text { for all } x \in \Omega\right\},
$$

and we can say $u$ is a refractor if $\tilde{F}_{u}\left(x_{0}\right) \neq \emptyset$ for all $x_{0} \in \Omega$. With this new definition, we can obtain the same regularity results as with definition (4.1) by changing the inequalities accordingly. We indicate the changes. In Definition 3.1, condition (3.1) is replaced by:

$$
\phi\left(x, Y_{\bar{X}}(\lambda), Z\right) \leq \max \{\phi(x, \bar{Y}, Z), \phi(x, \hat{Y}, Z)\}-C_{1}|\bar{Y}-\hat{Y}|^{2}|x-z|^{2} .
$$

Inequality (3.2) is replaced by:

$$
\left.\frac{d^{2}}{d \epsilon^{2}}\left\langle D^{2} \phi\left(x_{0}, Y_{\epsilon}, X_{0}\right) \eta, \eta\right\rangle\right|_{\epsilon=0} \geq C|\xi|^{2}|\eta|^{2}
$$

Lemma 4.1 is replaced by the convexity of $u$. The inequality (4.2) is replaced by: (7.4) $u(x)-\phi\left(x, Y, X_{0}^{\star}\right) \geq-C_{0}|\bar{Y}-\hat{Y}||\bar{x}-\hat{x}|-C_{1}|Y(\lambda)-Y|\left|x-x_{0}\right|+C_{2}|\bar{Y}-\hat{Y}|^{2}\left|x-x_{0}\right|^{2}$, and in the proof of Lemma 4.2 , min is replaced by max with the corresponding changes in the inequalities. For the example in Section 6, we now get that condition (6.3) is replaced by

$$
\frac{\kappa}{1-\kappa}>\psi(0)\left\langle D^{2} \psi(0) \xi, \xi\right\rangle,
$$

for all unit vectors $\xi$.

\section{REgUlARITY RESULTS FOR THE PARALLEL REFLECTOR PROBLEM IN THE NEAR FIELD}

CASE

In this section we shall prove results that are similar to the ones proved in the previous sections but for the reflector problem. Since the arguments are similar, we will omit most details.

8.1. Reflection. We first review the process of reflection. Our setting is $\mathbb{R}^{n+1}$, and points will be denoted by $X=\left(x, x_{n+1}\right)$. We consider parallel rays moving in the direction $e_{n+1}$. Let $T$ be a hyperplane in $\mathbb{R}^{n+1}$ with upper unit normal $N$ and let $X \in T$. By Snell law of reflection, a ray coming from below with direction $e_{n+1}$ that hits $T$ at $X$ is reflected in the unit direction $\Lambda=e_{n+1}-2\left(e_{n+1} \cdot N\right) N$. In particular, if $v \in \mathbb{R}^{n}$ and $N=\frac{(-v, 1)}{\left(1+|v|^{2}\right)^{\frac{1}{2}}}$, then the reflected direction is the unit vector

$$
\Lambda(v)=\left(\frac{2 v}{1+|v|^{2}}, \frac{|v|^{2}-1}{1+|v|^{2}}\right) .
$$

The reflected ray consists of the points $Y=X+s \Lambda$, for $s>0$. We have in mind here that $v=D u(x)$ and $X=(x, u(x))$, where $u$ is a reflector. 
If $b>0$, and $Y \in \mathbb{R}^{n+1}$, then the set of $X \in \mathbb{R}^{n+1}$ with $|X-Y|+x_{n+1}-y_{n+1}=b$ is a downwards paraboloid with focus at $Y$. It can be written as the graph of the function $p(x, Y)=y_{n+1}+\frac{b^{2}-|x-y|^{2}}{2 b}$. The ray with direction $e_{n+1}$ that hits the graph of $p$ at $X=(x, p(x, Y))$ is reflected in direction $Y-X$. If $Y, X_{0} \in \mathbb{R}^{n+1}$ with $X_{0}$ not in the vertical ray with direction $-e_{n+1}$ emanating from $Y$, then there exists a unique paraboloid with focus at $Y$ passing through $X_{0}$. Such a paraboloid is described by the function $p\left(x, Y, X_{0}\right)=y_{n+1}+\frac{b^{2}-|x-y|^{2}}{2 b}$ where $b=\left|X_{0}-Y\right|+x_{0_{n+1}}-y_{n+1}$. We will frequently use the following fact:

if the focus $Y$ of the paraboloid defined by $p\left(x, Y, X_{0}\right)$ satisfies $Y=X_{0}+s \Lambda(v)$ for some $s>0$ and $v \in \mathbb{R}^{n}$, then $D_{x} p\left(x_{0}, Y, X_{0}\right)=v$,

where $\Lambda(v)$ is given by (8.1).

8.2. Set up. Let $\Omega \subset \mathbb{R}^{n}$ be open and bounded, and let $C_{\Omega}$ be the cylinder $C_{\Omega}=$ $\Omega \times[0, M]$. For a fixed number $\beta>0$ we define the region

$$
\mathcal{T}=\left\{Y \in R^{n+1}:|X-Y|+x_{n+1}-y_{n+1} \geq \beta \text { for all } X \in C_{\Omega}\right\} .
$$

The set $\mathcal{T}$ consists of the points $Y$ such that the cylinder $C_{\Omega}$ is contained outside the interior of the paraboloid $|X-Y|+x_{n+1}-y_{n+1}=\beta$. We will assume that the target $\Sigma$ has convex hull bounded and contained in $\mathcal{T}$.

Proceeding as in Subsection 2.4 it is easy to see that $\left|\frac{\partial p}{\partial x_{0_{n+1}}}\left(x, Y, X_{0}\right)\right|,\left|\frac{\partial^{2} p}{\partial x_{i} \partial y_{j}}\left(x, Y, X_{0}\right)\right|$ are bounded uniformly for all $x \in \Omega, Y \in K \subset \mathcal{T}$, and $X_{0} \in C_{\Omega}$ for $1 \leq i \leq n$ and $1 \leq j \leq n+1$, where $K$ is compact. Hence as in Lemmas 2.2 and 2.3, we obtain the following.

Lemma 8.1. If $X_{0} \in C_{\Omega}$ with $X_{0}+h e_{n+1} \in C_{\Omega}$, and $Y, \bar{Y} \in \Sigma$, then we have

$$
\left|p\left(x, Y, X_{0}\right)-p\left(x, Y, X_{0}+h e_{n+1}\right)\right| \leq C|h|,
$$

and

for all $x \in \Omega$.

$$
\left|p\left(x, Y, X_{0}\right)-p\left(x, \bar{Y}, X_{0}\right)\right| \leq C\left|x-x_{0} \| Y-\bar{Y}\right|
$$

8.3. Hypothesis on the target set. Given $\bar{Y}, \hat{Y} \in \Sigma, X_{0} \in C_{\Omega}$, we let $\bar{v}=D_{x} p\left(x_{0}, \bar{Y}, X_{0}\right)$, $\hat{v}=D_{x} p\left(x_{0}, \hat{Y}, X_{0}\right)$, and $v(\lambda)=(1-\lambda) \bar{v}+\lambda \hat{v}$, for $\lambda \in[0,1]$. Consider the set of points

$$
C\left(X_{0}, \bar{Y}, \hat{Y}\right)=\left\{Y=X_{0}+s \Lambda(v(\lambda)): s>0, \lambda \in[0,1]\right\},
$$

where $\Lambda$ is given by (8.1). Notice that from (8.2), if $Y=X_{0}+s \Lambda(v(\lambda))$, then $D_{x} p\left(x_{0}, Y, X_{0}\right)=v(\lambda)$.

For $X_{0} \in C_{\Omega}$ and for $\bar{Y}, \hat{Y} \in \Sigma$ we assume $[\bar{Y}, \hat{Y}]_{X_{0}}:=C\left(X_{0}, \bar{Y}, \hat{Y}\right) \cap \Sigma$ is a curve joining $\bar{Y}$ and $\hat{Y}$.

We introduce the following condition on the target $\Sigma$, similar to Definition 3.1 for refractors. 
Definition 8.2. If $X_{0} \in C_{\Omega}$ we say that the target $\Sigma$ is regular from $X_{0}$ if there exists a neighborhood $U_{X_{0}}$ and positive constants $C_{X_{0}}$, depending on $U_{X_{0}}$, such that for all $\bar{Y}, \hat{Y} \in \sum$ and $Z=\left(z, z_{n+1}\right) \in U_{X_{0}}$ we have

$$
\max \{p(x, \bar{Y}, Z), p(x, \hat{Y}, Z)\} \geq p\left(x, Y_{Z}(\lambda), Z\right)+C_{X_{0}}|\bar{Y}-\hat{Y}|^{2}|x-z|^{2}
$$

for all $x \in \Omega, 1 / 4 \leq \lambda \leq 3 / 4$, and $Y_{Z}(\lambda)=Z+s_{Z}(\Lambda(v(\lambda))) \Lambda(v(\lambda))$. Here $\bar{v}=$ $D_{x} \phi(z, \bar{Y}, Z), \hat{v}=D_{x} \phi(z, \hat{Y}, Z)$, and $v(\lambda)=(1-\lambda) \bar{v}+\lambda \hat{v}$.

As in the case of refractors, we also have a differential condition that is equivalent to (8.3). This is the contents of the following theorem.

Theorem 8.3. Suppose that there exists a constant $C$ such that for all $\xi$ and $\eta$, perpendicular vectors in $R^{n}$, and for $X_{0} \in C_{\Omega}$ and for $Y_{0} \in \Sigma$, we have

$$
\left.\frac{d^{2}}{d \epsilon^{2}}\left\langle D_{x}^{2} p\left(x_{0}, Y_{\epsilon}, X_{0}\right) \eta, \eta\right\rangle\right|_{\epsilon=0} \geq C|\xi|^{2}|\eta|^{2}
$$

where, $v_{0}=D p\left(x_{0}, Y_{0}, X_{0}\right)$ and $Y_{\epsilon}=X_{0}+s_{X_{0}}\left(\Lambda\left(v_{0}+\epsilon \xi\right)\right) \Lambda\left(v_{0}+\epsilon \xi\right)$.

Then there exists a structural constant $C$ such that for $\bar{Y}, \hat{Y} \in \Sigma$ and $X_{0} \in C_{\Omega}$ we have for $\lambda \in[1 / 4,3 / 4]$ and for all $x \in \Omega$ that

$$
\max \left\{p\left(x, \bar{Y}, X_{0}\right), p\left(x, \hat{Y}, X_{0}\right)\right\} \geq p\left(x, Y_{X_{0}}(\lambda), X_{0}\right)+C|\bar{Y}-\hat{Y}|^{2}\left|x-x_{0}\right|^{2}
$$

Conversely, (8.5) implies (8.4).

Proof. That (8.5) implies (8.4), follows in the same way as (3.3) implies (3.2) in Theorem 3.2.

We first show that if (8.4) holds for $\xi \perp \eta$, then it holds for all vectors $\xi, \eta$. In fact, we have

$$
\left\langle D_{x}^{2} p\left(x_{0}, Y_{\epsilon}, X_{0}\right) \eta, \eta\right\rangle=-\frac{|\eta|^{2}}{2} \frac{1+\left|v_{0}+\epsilon \xi\right|^{2}}{s_{X_{0}}\left(\Lambda\left(v_{0}+\epsilon \xi\right)\right)}
$$

So if $\xi \cdot \eta \neq 0$, we pick $\eta^{\prime}$ with $\left|\eta^{\prime}\right|=|\eta|$ and $\eta^{\prime} \cdot \xi=0$, and we have $\left\langle D_{x}^{2} p\left(x_{0}, Y_{\epsilon}, X_{0}\right) \eta, \eta\right\rangle=$ $\left\langle D_{x}^{2} p\left(x_{0}, Y_{\epsilon}, X_{0}\right) \eta^{\prime}, \eta^{\prime}\right\rangle$. Let $\bar{Y}, \hat{Y} \in \sum$ and $X_{0} \in C_{\Omega}, \bar{v}=D p\left(x_{0}, \bar{Y}, X_{0}\right), \hat{v}=D p\left(x_{0}, \hat{Y}, X_{0}\right)$, $\xi=\hat{v}-\bar{v}$, and $Y_{X_{0}}(\lambda)=X_{0}+s_{X_{0}}(\Lambda(\bar{v}+\lambda \xi)) \Lambda(\bar{v}+\lambda \xi)$. We then have

$$
\frac{d}{d \lambda^{2}}\left\langle D_{x}^{2} p\left(x_{0}, Y_{X_{0}}(\lambda), X_{0}\right) \eta, \eta\right\rangle \geq C|\xi|^{2}|\eta|^{2}
$$

for $0 \leq \lambda \leq 1$. Fix $x \in \Omega$, and let $f(\lambda)=\left\langle D_{x}^{2} p\left(x_{0}, Y_{X_{0}}(\lambda), X_{0}\right)\left(x-x_{0}\right),\left(x-x_{0}\right)\right\rangle$. Since $f^{\prime \prime}(\lambda) \geq C|\xi|^{2}\left|x-x_{0}\right|^{2} \geq C|\bar{Y}-\hat{Y}|^{2}\left|x-x_{0}\right|^{2}$, where the second inequality follows from the analogue of (2.12) for reflectors, it follows that $(1-\lambda) f(0)+\lambda f(1) \geq$ 


$$
\begin{aligned}
& f(\lambda)+C \lambda(1-\lambda)\left|x-x_{0}\right|^{2}|\bar{Y}-\hat{Y}|^{2} \text {. Therefore, } \\
& \max \left\{p\left(x, \bar{Y}, X_{0}\right), p\left(x, \hat{Y}, X_{0}\right)\right\}-p\left(x, Y_{X_{0}}(\lambda), X_{0}\right) \\
& \geq(1-\lambda) p\left(x, \bar{Y}, X_{0}\right)+\lambda p\left(x, \hat{Y}, X_{0}\right)-p\left(x, Y_{X_{0}}(\lambda), X_{0}\right) \\
& =\frac{1}{2}\left\langle\left((1-\lambda) D_{x}^{2} p\left(x_{0}, \bar{Y}, X_{0}\right)+\lambda D_{x}^{2} p\left(x_{0}, \hat{Y}, X_{0}\right)-D_{x}^{2} p\left(x_{0}, Y_{X_{0}}(\lambda), X_{0}\right)\right)\left(x-x_{0}\right), x-x_{0}\right\rangle \\
& \geq C \lambda(1-\lambda)\left|x-x_{0}\right|^{2}|\bar{Y}-\hat{Y}|^{2},
\end{aligned}
$$

for all $x \in \Omega$, and $0 \leq \lambda \leq 1$. This completes the proof of the theorem.

Remark 8.4. Analogously to Remark 3.3, a local version of 8.4 can be stated as follows: The target $\Sigma$ is regular from $X_{0} \in C_{\Omega}$ if there exists a neighborhood $U_{X_{0}}$ and a constant $C$ depending on $X_{0}$ such that for all $Y_{0} \in \Sigma$ and for all $Z \in U_{X_{0}}$ and for all vectors $\xi$ and $\eta$ such that $\xi \perp \eta$ we have

$$
\left.\frac{d^{2}}{d \epsilon^{2}}\left\langle D_{x}^{2} p\left(z, Y_{\epsilon}, Z\right) \eta, \eta\right\rangle\right|_{\epsilon=0} \geq C|\xi|^{2}|\eta|^{2}
$$

where $Y_{\epsilon}=Z+s(\Lambda(v+\epsilon \xi)) \Lambda(v+\epsilon \xi)$ and $v=D p\left(z, Y_{0}, Z\right)$ (8.3).

Following the proof of Theorem 8.3, one can show that (8.7) is equivalent to

8.4. Definition of parallel reflector and hypothesis on the measures. We say $u: \Omega \rightarrow[0, M]$ is a parallel reflector from $\Omega$ to $\Sigma$ if for each $x_{0} \in \Omega$, there exists $Y \in \Sigma$ such that $u(x) \geq p\left(x, Y, X_{0}\right)$ for all $x \in \Omega$, where $X_{0}=\left(x_{0}, u\left(x_{0}\right)\right)$. In this case, we say $Y \in F_{u}\left(x_{0}\right)$. Any reflector is Lipschitz in $\Omega$ with a uniform Lipschitz constant depending on the bounds for the derivatives of $p$, which are uniform for $Y \in K \Subset \mathcal{T}, x \in \Omega$ and $X_{0} \in C_{\Omega}$.

Existence of solutions with this definition of parallel reflector can be proved in a way similar to the existence of parallel refractors as done in [?]. We omit the corresponding details.

We make the following hypothesis on the measures.

Also similarly to (5.1), we introduce the following local condition at $X_{0} \in C_{\Omega}$ between the measure $\sigma$ and target $\Sigma$ : There exist a neighborhood $U_{X_{0}}$ and a constant $\hat{C}>0$ depending on $X_{0}$ such that

$$
\sigma\left(N_{\mu}\left(\left\{[\bar{Y}, \hat{Y}]_{Z}: \lambda \in[1 / 4,3 / 4]\right\}\right) \cap \Sigma\right) \geq \hat{C} \mu^{n-1}|\bar{Y}-\hat{Y}|
$$

for any $\bar{Y}, \hat{Y} \in \Sigma, Z \in U_{X_{0}}$ and for all $\mu>0$ small (depending on $X_{0}$ ). Here $N_{\mu}(E)$ denotes the $\mu$ - neighborhood of the set $E$ in $R^{n+1}$.

The following lemma is the analogue of Lemma 4.1 .

Lemma 8.5. If $u$ is a parallel reflector, then for any $\bar{x}, \hat{x} \in \Omega$ and for any $s \in[0,1]$ we have $u((1-s) \bar{x}+s \hat{x}) \leq(1-s) u(\bar{x})+s u(\hat{x})+C|\bar{x}-\hat{x}|^{2} s(1-s)$, where $C$ is a structural constant.

The following lemma is the analogue of Lemma 4.2 . 
Lemma 8.6. Let $u$ be a parallel reflector such that the target $\Sigma$ is regular from $X^{\star}=$ $\left(x^{\star}, u\left(x^{\star}\right)\right)$ in the sense of Definition 8.2. There exists constants $\delta$ and $C_{1}$, depending on $X^{\star}$ such that if $\bar{x}, \hat{x} \in B_{\delta}\left(x^{\star}\right)$ and $\bar{Y} \in F_{u}(\bar{x}), \hat{Y} \in F_{u}(\hat{x})$ and $|\bar{Y}-\hat{Y}| \geq|\bar{x}-\hat{x}|$; then there exists $x_{0} \in \overline{\bar{x} \hat{x}}$ such that if $X_{0}^{\star}=\left(x_{0}, u\left(x_{0}\right)\right)$, then we have for all $Y(\lambda) \in[\bar{Y}, \hat{Y}]_{X_{0}^{\star}}$ and for all $Y \in \Sigma$ and for all $x \in \Omega$ that the following inequality holds

$$
u(x)-p\left(x, Y, X_{0}^{\star}\right) \geq-C|\bar{Y}-\hat{Y}||\bar{x}-\hat{x}|-C|Y(\lambda)-Y|\left|x-x_{0}\right|+C_{1} \lambda(1-\lambda)|\bar{Y}-\hat{Y}|^{2}\left|x-x_{0}\right|^{2} .
$$
We remark that $C$ is a structural constant.

Proof. The proof is very much the same as in the refractor problem. We indicate the main points.

Since $\Sigma$ is regular from $X^{\star}$, there exists a neighborhood $U_{X^{\star}}$ of $X^{\star}$ such that (8.3) holds for all $\bar{Y}, \hat{Y} \in \Sigma$ and all $Z \in U_{X^{\star}}$. Since parallel reflectors are uniformly Lipschitz in $\Omega$, there exists $\delta>0$ such that $(x, u(x)) \in U_{X^{\star}}$ for all $x \in B_{\delta}\left(x^{\star}\right)$. For $x \in \Omega$ we have $u(x) \geq \max \{p(x, \bar{Y}, \bar{X}), p(x, \hat{Y}, \hat{X})\}$, and there exists $x_{0} \in[\bar{x}, \hat{x}]$ such that $p\left(x_{0}, \bar{Y}, \bar{X}\right)=p\left(x_{0}, \hat{Y}, \hat{X}\right):=x_{0_{n+1}}$. Set $X_{0}=\left(x_{0}, x_{0_{n+1}}\right)$, and $X_{0}^{\star}=\left(x_{0}, u\left(x_{0}\right)\right) \in U_{X^{\star}}$ and notice that $u\left(x_{0}\right) \geq x_{0_{n+1}}$. Similarly as in Lemma 4.2 we get

$$
\begin{aligned}
u(x) & \geq \max \{p(x, \bar{Y}, \bar{X}), p(x, \hat{Y}, \hat{X})\}=\max \left\{p\left(x, \bar{Y}, X_{0}\right), p\left(x, \hat{Y}, X_{0}\right)\right\} \\
& =\max \left\{p\left(x, \bar{Y}, X_{0}^{\star}\right), p\left(x, \hat{Y}, X_{0}^{\star}\right)\right\}-E .
\end{aligned}
$$

Using Lemmas 8.1 and 8.5, and proceeding as in the proof of claim (4.3), we get $0 \leq E \leq C\left(u\left(x_{0}\right)-x_{0_{n+1}}\right) \leq C|\bar{Y}-\hat{Y} \| \bar{x}-\hat{x}|$. Hence $\max \left\{p\left(x, \bar{Y}, X_{0}^{\star}\right), p\left(x, \hat{Y}, X_{0}^{\star}\right)\right\}-E \geq$ $\max \left\{p\left(x, \bar{Y}, X_{0}^{\star}\right), p\left(x, \hat{Y}, X_{0}^{\star}\right)\right\}-C|\bar{Y}-\hat{Y}||\bar{x}-\hat{x}| \geq p\left(x, Y_{X_{0}^{\star}}(\lambda), X_{0}^{\star}\right)+C_{1} \lambda(1-\lambda)|\bar{Y}-\hat{Y}|^{2} \mid x-$ $\left.x_{0}\right|^{2}-C|\bar{Y}-\hat{Y}||\bar{x}-\hat{x}|$, where we have used (8.3). Now using the second inequality in Lemma 8.1, we get $p\left(x, Y_{X_{0}^{\star}}(\lambda), X_{0}^{\star}\right)+C_{1} \lambda(1-\lambda)|\bar{Y}-\hat{Y}|^{2}\left|x-x_{0}\right|^{2}-C|\bar{Y}-\hat{Y}||\bar{x}-\hat{x}| \geq$ $p\left(x, Y, X_{0}^{\star}\right)-C\left|x-x_{0}\right||Y-Y(\lambda)|+C_{1} \lambda(1-\lambda)|\bar{Y}-\hat{Y}|^{2}\left|x-x_{0}\right|^{2}-C|\bar{Y}-\hat{Y}||\bar{x}-\hat{x}|$, which proves the lemma.

We then obtain results similar to Theorems 4.5 and 5.1 for parallel reflectors.

Theorem 8.7. Suppose $u$ is a parallel reflector, the target $\Sigma$ is regular from $X^{\star}=$ $\left(x^{\star}, u\left(x^{\star}\right)\right)$ in the sense of Definition 8.2. There exist a ball $B_{\delta}\left(x^{\star}\right) \subset \Omega$, and a constant $M>0$ depending on $X^{\star}$ such that if $\hat{x}, \bar{x} \in B_{\delta}\left(x^{\star}\right)$ and $\bar{Y} \in F_{u}(\bar{x}), \hat{Y} \in F_{u}(\hat{x})$ are such that

$$
\frac{|\bar{Y}-\hat{Y}|}{|\bar{x}-\hat{x}|} \geq \max \left\{1,\left(\frac{M}{\operatorname{dist}\left(B_{\delta}\left(x^{\star}\right), \partial \Omega\right)}\right)^{2}\right\},
$$

then there exists $x_{0} \in[\bar{x}, \hat{x}]$ such that, setting $X_{0}^{\star}=\left(x_{0}, u\left(x_{0}\right)\right)$, we have

$$
N_{\mu}\left(\left\{Y(\lambda) \in[\bar{Y}, \hat{Y}]_{X_{0}^{\star}}: \lambda \in[1 / 4,3 / 4]\right\}\right) \cap \Sigma \subseteq F_{u}\left(B_{\eta}\left(x_{0}\right)\right)
$$

with $\mu=|\bar{Y}-\hat{Y}|^{\frac{3}{2}}|\bar{x}-\hat{x}|^{\frac{1}{2}}$ and $\eta=M \frac{|\bar{x}-\hat{x}|^{\frac{1}{2}}}{|\bar{Y}-\hat{Y}|^{\frac{1}{2}}}$. 
Theorem 8.8. Suppose $u$ is a parallel reflector, the target $\Sigma$ is regular from $X^{\star}=$ $\left(x^{\star}, u\left(x^{\star}\right)\right)$ in the sense of Definition 8.2. and there exist constants $C_{0}>0$ and $1 \leq q<$ $\frac{n}{n-1}$ such that

$$
\sigma\left(F_{u}\left(B_{\eta}\right)\right) \leq C_{0} \eta^{n / q}
$$

for all balls $B_{\eta} \subseteq \Omega$. Suppose in addition that the local condition (8.8) is satisfied at $X^{\star}$.

Then there exist a ball $B_{\delta}\left(x^{\star}\right) \subset \Omega$, and a constant $M>0$ depending on $X^{\star}$, such that if $\hat{x}, \bar{x} \in B_{\delta}\left(x^{\star}\right), \bar{Y} \in F_{u}(\bar{x}), \hat{Y} \in F_{u}(\hat{x})$ are such that

$$
\frac{|\bar{Y}-\hat{Y}|}{|\bar{x}-\hat{x}|} \geq \max \left\{1,\left(\frac{M}{\operatorname{dist}\left(B_{\delta}\left(x^{\star}\right), \partial \Omega\right)}\right)^{2}\right\},
$$

then we have $|\bar{Y}-\hat{Y}| \leq C_{1}|\bar{x}-\hat{x}|^{\alpha}$ with $\alpha=\frac{\frac{n}{2 q}-\frac{n-1}{2}}{1+\frac{3}{2}(n-1)+\frac{n}{2 q}}$, where $C_{1}$ depends only on $C_{0}$ and $\hat{C}$ in 8.8 , and therefore from $X^{\star}$.

We can then proceed in exactly the same way as in the refractor problem to get the analogues of Theorem 5.2, and Corollary 5.3 for parallel reflectors.

Remark 8.9. We show here that when the target $\Sigma$ is contained in a non-vertical hyperplane, then condition (8.4) holds. Notice that from (8.6), it is sufficient to show that

$$
\left\langle D_{v}^{2}\left(\frac{1+|v|^{2}}{s_{X}(\Lambda(v))}\right) \xi, \xi\right\rangle \leq-C
$$

for all $v \in \mathbb{R}^{n}$ and $|\xi|=1(|\eta|=1)$. Let $w \in \mathbb{R}^{n}$, and $\Sigma=\left\{\left(y, y_{n+1}\right), y_{n+1}=y \cdot w\right\}$. Suppose $0<C_{1} \leq x_{n+1}-x \cdot w \leq C_{2}$ for all $X \in C_{\Omega}$. We calculate $\frac{1+|v|^{2}}{s_{X}(\Lambda(v))}$. From (8.1) we have

$$
\begin{aligned}
y-x & =s_{X}(\Lambda(v)) \frac{2 v}{1+|v|^{2}} \\
y_{n+1}-x_{n+1} & =s_{X}(\Lambda(v)) \frac{|v|^{2}-1}{1+|v|^{2}} .
\end{aligned}
$$

Hence $\frac{1+|v|^{2}}{s_{X}(\Lambda(v))}=\frac{2 v \cdot w+1-|v|^{2}}{x_{n+1}-x \cdot w}$, and then $\left\langle D_{v}^{2}\left(\frac{1+|v|^{2}}{s_{X}(\Lambda(v))}\right) \xi, \xi\right\rangle=-\frac{2}{x_{n+1}-x \cdot w} \leq$ $-C$.

If $\left(y, y_{n+1}\right) \in \Sigma$ and $\Sigma$ is a vertical plane, then $y \cdot w=0$ and we get $\frac{1+|v|^{2}}{s_{X}(\Lambda(v))}=$ $-\frac{2 v \cdot w}{x \cdot w}$, so $D_{v}^{2}\left(\frac{1+|v|^{2}}{s_{X}(\Lambda(v))}\right)=0$. 
8.5. On the definition of reflector. When rays emanate from a source point, the definition of reflector (near field and far field) given in the literature, is made with paraboloids or ellipsoids touching the reflector from above, i.e., supporting paraboloids or ellipsoids enclosing the reflector. With such definition, the class of reflectors is restricted to strictly concave functions and therefore, for instance, a plane cannot be a reflector. However, in the applications, planes are typical examples of reflectors. To cover this case, we have chosen to define in Subsection 8.4 parallel reflector with paraboloids touching from below, see also Subsection 8.10 for results with a definition of parallel reflector with paraboloids touching from above.

We will illustrate with simple examples that both type of solutions exist for a particular problem. Let us consider in one dimension the following problem: Find a reflector $u$ so that collimated rays emanating from the interval $[-1,1]$ in the direction $(0,1)$ are reflected into the interval $[2,4]$, also on the $x$-axis, and such that Lebesgue measure is preserved. For each $x \in[-1,1]$, let $y=y(x) \in[2,4]$ be the reflection map. Then by the law of reflection, the reflector $u$ satisfies the equation

$$
u^{\prime}(x)=\frac{y-x}{\left((y-x)^{2}+u(x)^{2}\right)^{1 / 2}+u(x)} .
$$

Since $y>x$, we have that $u$ increases. The only maps $y:[-1,1] \rightarrow[2,4]$ that preserve Lebesgue measure and are $C^{1}$ are $y(x)=x+3$ and $y(x)=-x+3$.

Let us first take $y(x)=x+3$, hence $u$ satisfies the equation $u^{\prime}(x)=\frac{3}{\left(9+u(x)^{2}\right)^{1 / 2}+u(x)}$.

Let $a \geq 0$ and consider the solution $u$ with initial condition $u(-1)=a ; u$ is increasing and concave. So $u \geq 0$ on $[-1,1]$. We claim that $u$ is a solution that can be touched locally from below with paraboloids, and globally, also from below, if $a$ is sufficiently large. Therefore $u$ cannot be touched from above with paraboloids. In fact, we first show that it can be touched from below locally. Fix $x_{0} \in(-1,1)$, let $X_{0}=\left(x_{0}, u\left(x_{0}\right)\right)$, and $Y_{0}=\left(x_{0}+3,0\right)$. With the definition of $p$ given in Subsection 8.1. we have $p\left(x, Y_{0}, X_{0}\right)=u\left(x_{0}\right)+\frac{9-\left|x-y_{0}\right|^{2}}{2\left(\left(u\left(x_{0}\right)^{2}+9\right)^{1 / 2}+u\left(x_{0}\right)\right)}$, and simplifying we get

$$
\begin{aligned}
p\left(x, Y_{0}, X_{0}\right) & =u\left(x_{0}\right)+\frac{3\left(x-x_{0}\right)}{\left(u\left(x_{0}\right)^{2}+9\right)^{1 / 2}+u\left(x_{0}\right)}-\frac{\left(x-x_{0}\right)^{2}}{2\left(\left(u\left(x_{0}\right)^{2}+9\right)^{1 / 2}+u\left(x_{0}\right)\right)} \\
& =u\left(x_{0}\right)+u^{\prime}\left(x_{0}\right)\left(x-x_{0}\right)-\frac{\left(x-x_{0}\right)^{2}}{2\left(\left(u\left(x_{0}\right)^{2}+9\right)^{1 / 2}+u\left(x_{0}\right)\right)}
\end{aligned}
$$

To have that $u(x) \geq p\left(x, Y_{0}, X_{0}\right)$ locally, we then need that $u^{\prime \prime}\left(x_{0}\right)>\frac{-1}{\left(u\left(x_{0}\right)^{2}+9\right)^{1 / 2}+u\left(x_{0}\right)}$. Using the equation for $u$, we get $u^{\prime \prime}\left(x_{0}\right)=\frac{-9}{\left(\left(u\left(x_{0}\right)^{2}+9\right)^{1 / 2}+u\left(x_{0}\right)\right)^{2}\left(u\left(x_{0}\right)^{2}+9\right)^{1 / 2}}$ and therefore $u^{\prime \prime}\left(x_{0}\right)>\frac{-1}{\left(u\left(x_{0}\right)^{2}+9\right)^{1 / 2}+u\left(x_{0}\right)}$, since $u \geq 0$. 
In order to get that $u(x) \geq p\left(x, Y_{0}, X_{0}\right)$ for all $x \in[-1,1]$, we can take $a$ sufficiently large. To see this, since $(u-p)^{\prime}\left(x_{0}\right)=0$, and $(u-p)\left(x_{0}\right)=0$, it is enough to show that $(u-p)^{\prime \prime}(x) \geq 0$ for all $-1<x<1$. We have $u^{\prime \prime}(x) \geq \frac{-9}{a^{2}}$ for all $x \in[-1,1]$. Using that $u$ is increasing, concave, $u^{\prime} \leq 1$, and $u(-1)=a$, we get that $p^{\prime \prime}(x) \leq-\frac{1}{3 u\left(x_{0}\right)} \leq \frac{-1}{3(a+2)}$, for any $x, x_{0} \in[-1,1]$. Therefore, if $\frac{1}{3(a+2)}>9 / a^{2}$, then we obtain the desired conclusion.

On the other hand, the solutions corresponding to the map $y=-x+3$ satisfy the equation $u^{\prime}(x)=\frac{-2 x+3}{\left((-2 x+3)^{2}+u(x)^{2}\right)^{1 / 2}+u(x)}$. We will show that the solution $u$ can be enclosed by paraboloids.

Fix $x_{0} \in(-1,1)$, let $X_{0}=\left(x_{0}, u\left(x_{0}\right)\right)$, and $Y_{0}=\left(-x_{0}+3,0\right)$. Then $p\left(x, Y_{0}, X_{0}\right)=$ $u\left(x_{0}\right)+\frac{\left|3-2 x_{0}\right|^{2}-\left|x-\left(3-x_{0}\right)\right|^{2}}{2\left(\left(u\left(x_{0}\right)^{2}+\left(3-2 x_{0}\right)^{2}\right)^{1 / 2}+u\left(x_{0}\right)\right)}$ and we write

$$
\begin{aligned}
p\left(x, Y_{0}, X_{0}\right) & =u\left(x_{0}\right)+\frac{3\left(x-x_{0}\right)\left(3-2 x_{0}\right)}{\left(u\left(x_{0}\right)^{2}+\left(3-2 x_{0}\right)^{2}\right)^{1 / 2}+u\left(x_{0}\right)}-\frac{\left(x-x_{0}\right)^{2}}{2\left(\left(u\left(x_{0}\right)^{2}+\left(3-2 x_{0}\right)^{2}\right)^{1 / 2}+u\left(x_{0}\right)\right)} \\
& =u\left(x_{0}\right)+u^{\prime}\left(x_{0}\right)\left(x-x_{0}\right)-\frac{\left(x-x_{0}\right)^{2}}{2\left(\left(u\left(x_{0}\right)^{2}+\left(3-2 x_{0}\right)^{2}\right)^{1 / 2}+u\left(x_{0}\right)\right)} .
\end{aligned}
$$

To have that $u(x) \leq p\left(x, Y_{0}, X_{0}\right)$ locally, we then need that $u^{\prime \prime}\left(x_{0}\right)<\frac{-1}{\left(u\left(x_{0}\right)^{2}+\left(3-2 x_{0}\right)^{2}\right)^{1 / 2}+u\left(x_{0}\right)}$. We have

$$
u^{\prime \prime}(x)=-\frac{2 u(x)+\frac{(3-2 x)^{2}}{u(x)+\sqrt{(3-2 x)^{2}+u(x)^{2}}}}{(3-2 x)^{2}+u(x)^{2}+u(x) \sqrt{(3-2 x)^{2}+u(x)^{2}}}:=-\frac{1}{u(x)} F(u(x), x) .
$$

If $u\left(x_{0}\right) \geq 0$, then $u^{\prime \prime}\left(x_{0}\right)<\frac{-1}{\left(u\left(x_{0}\right)^{2}+\left(3-2 x_{0}\right)^{2}\right)^{1 / 2}+u\left(x_{0}\right)}$. Since $u \geq 0$ for $-1 \leq x \leq 1$, we have that $u$ is increasing and concave in $[-1,1]$.

In order to get that $u(x) \leq p\left(x, Y_{0}, X_{0}\right)$ for all $x \in[-1,1]$, we will again take $a$ sufficiently large. To see this, since $(u-p)^{\prime}\left(x_{0}\right)=0$, and $(u-p)\left(x_{0}\right)=0$, it is enough to show that $(u-p)^{\prime \prime}(x) \leq 0$ for all $-1<x<1$. We first claim that there exists $a_{0}>0$ such that if $a>a_{0}$, and $u(-1)=a$, then

$$
u^{\prime \prime}(x) \leq-\frac{3}{4 u(x)}, \quad \forall x \in[-1,1] .
$$

Indeed, we have $\lim _{\lambda \rightarrow+\infty} F(\lambda, x)=1$ uniformly for $x \in[-1,1]$. Since $u$ is increasing and $u(-1)=a$, the claim follows. On the other hand, since $u$ is concave, $\left|u^{\prime}\right| \leq 1$, then $u(x) \leq u(-1)+u^{\prime}(-1)(x+1) \leq a+2$. Also $p^{\prime \prime}(x) \geq-\frac{1}{2 a}$. Then $u(x) p^{\prime \prime}(x) \geq$ $(a+2) p^{\prime \prime}(x) \geq-\frac{a+2}{2 a}$. Choosing $a$ sufficiently large with $\frac{a+2}{2 a}<3 / 4$, the desired result follows. 
In a similar vein, we consider the problem of finding a reflector reflecting the interval $[-1,1]$ on the $x$-axis into the interval $[2,16 / 3]$.

On $[-1,1]$ we take Lebesgue measure and on $[2,16 / 3]$ we take $\frac{3}{5}$ times Lebesgue measure. If the reflection mapping is $y=\frac{5}{3} x+\frac{11}{3}$, then the solution solves the equation $u^{\prime}(x)=\frac{\frac{2 x+11}{3}}{\left(\left(\frac{2 x+11}{3}\right)^{2}+u(x)^{2}\right)^{1 / 2}+u(x)}$. This equation has the plane solution $u(x)=\frac{1}{2} x+\frac{11}{4}$, which clearly cannot be touched with paraboloids from above.

Remark 8.10. Similarly to Section 7, we can define parallel reflector by taking paraboloids enclosing the solution. In other words, we can say $u: \Omega \rightarrow[0, M]$ is a parallel reflector from $\Omega$ to $\Sigma$ if for each $x_{0} \in \Omega$, there exists $Y \in \Sigma$ such that $u(x) \leq p\left(x, Y, X_{0}\right)$ for all $x \in \Omega$, where $X_{0}=\left(x_{0}, u\left(x_{0}\right)\right)$. The same regularity results hold with this definition of solution by changing accordingly the inequalities in the conditions on the target. Indeed, with the notation in Subsection 8.3, condition (8.3) is replaced by

$$
\min \{p(x, \bar{Y}, Z), p(x, \hat{Y}, Z)\} \leq p\left(x, Y_{Z}(\lambda), Z\right)-C_{X_{0}}|\bar{Y}-\hat{Y}|^{2}|x-z|^{2} .
$$

The analogue of condition (8.4) is now

$$
\left.\frac{d^{2}}{d \epsilon^{2}}\left\langle D_{x}^{2} p\left(x_{0}, Y_{\epsilon}, X_{0}\right) \eta, \eta\right\rangle\right|_{\epsilon=0} \leq-C|\xi|^{2}|\eta|^{2}
$$

and as before this is equivalent to (8.13). The concavity of $u$ now replaces Lemma 8.5. the inequality in Lemma 8.6 is replaced by

$u(x)-p\left(x, Y, X_{0}^{\star}\right) \leq C|\bar{Y}-\hat{Y}||\bar{x}-\hat{x}|+C|Y(\lambda)-Y|\left|x-x_{0}\right|-C_{1} \lambda(1-\lambda)|\bar{Y}-\hat{Y}|^{2}\left|x-x_{0}\right|^{2}$, and in the proof the max is replaced by the min. In contrast with Remark 8.9 , in this case a target contained in a hyperplane cannot satisfy (8.13).

Department of Mathematics, Temple University, Philadelphia, PA 19122

E-mail address: gutierre@temple.edu

Instituto Argentino de Matemática A. P. Calderón, CONiCet, Saavedra 15, Buenos Aires (CP 1083), Argentina

E-mail address: fedeleti@aim.com 